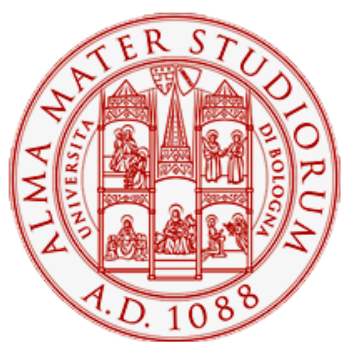

Alma Mater Studiorum - Università di Bologna DEPARTMENT OF ECONOMICS

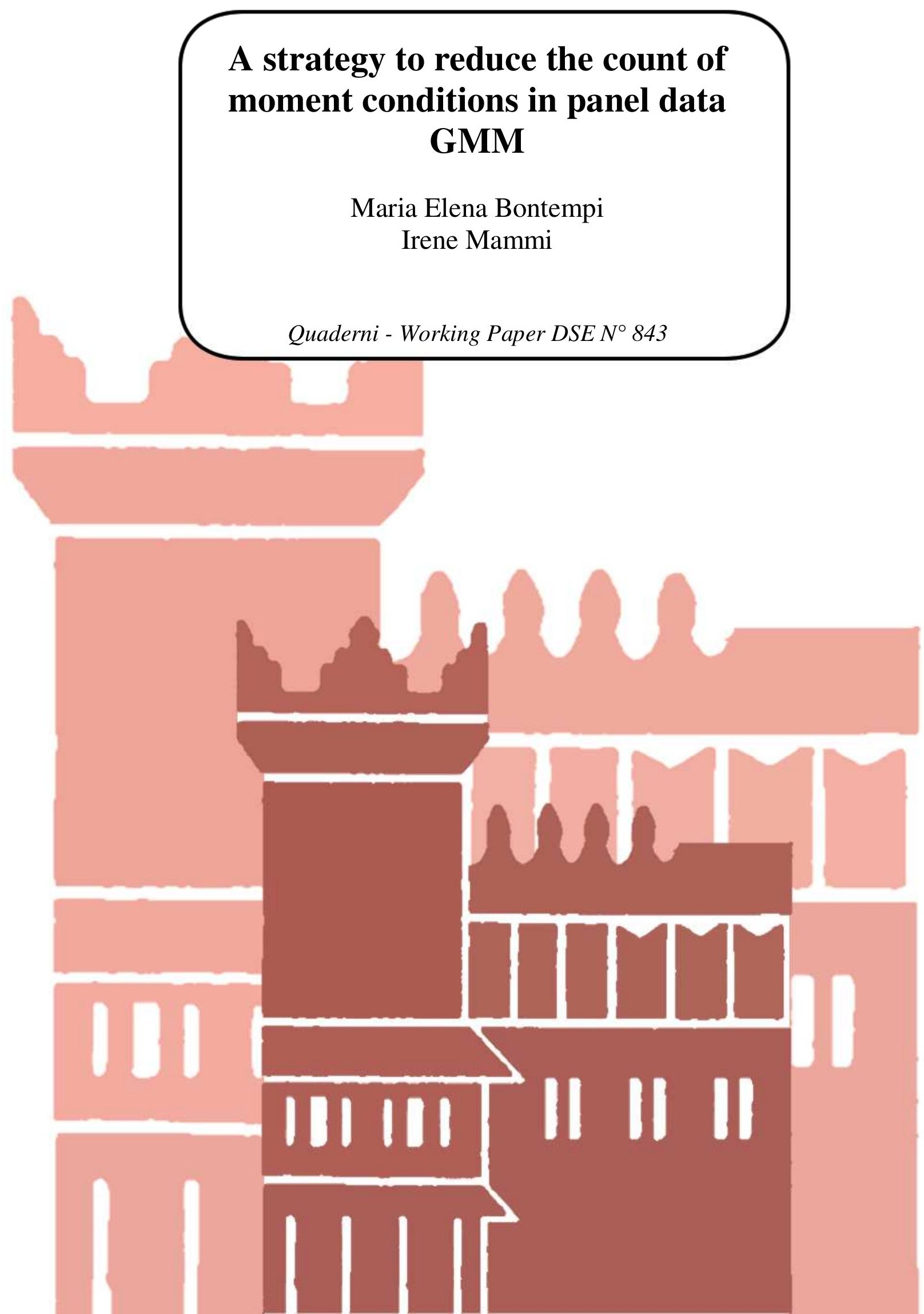




\title{
A strategy to reduce the count of moment conditions in panel data $\mathrm{GMM}^{*}$
}

\author{
Maria Elena Bontempit \\ Irene Mammi ${ }^{\ddagger}$
}

August 16, 2012

\begin{abstract}
The problem of instrument proliferation and its consequences (overfitting of endogenous variables, bias of estimates, weakening of Sargan/Hansen test) are well known. The literature provides little guidance on how many instruments is too many. It is common practice to report the instrument count and to test the sensitivity of results to the use of more or fewer instruments. Strategies to alleviate the instrument proliferation problem are the lag-depth truncation and/or the collapse of the instrument set (the latter being an horizontal squeezing of the instrument matrix). However, such strategies involve either a certain degree of arbitrariness (based on the ability and the experience of the researcher) or of trust in the restrictions implicitly imposed (and hence untestable) on the instrument matrix. The aim of the paper is to introduce a new strategy to reduce the instrument count. The technique we propose is statistically founded and purely datadriven and, as such, it can be considered a sort of benchmark solution to the problem of instrument proliferation. We apply the principal component analysis (PCA) on the instrument matrix and exploit the PCA scores as the instrument set for the panel generalized method-of-moments (GMM) estimation. Through extensive Monte Carlo simulations, under alternative characteristics of persistence of the endogenous variables, we compare the performance of the Difference GMM, Level and System GMM estimators
\end{abstract}

*The authors would like to thank Jerry Hausman, Jacques Mairesse, Whitney Newey and participants in the 18th International Panel Data Conference for comments and encouragement. The authors also thank Roberto Golinelli and Carla Rampichini for suggestions. The usual disclaimer applies.

${ }^{\dagger}$ Dep. of Economics, University of Bologna. Email: mariaelena.bontempi@unibo.it

‡Dep. of Economics, University of Bologna. Email: irene.mammi@unibo.it 
when lag truncation, collapsing and our principal component-based IV reduction (PCIVR henceforth) are applied to the instrument set. The same comparison has been carried out with two empirical applications on real data: the first replicates the estimates of Blundell and Bond [1998]; the second exploits a new and large panel data-set in order to assess the role of tangible and intangible capital on productivity. Results show that PCIVR is a promising strategy of instrument reduction.

JEL classification: C13, C15, C33, C36, C63.

Keywords: Panel data, generalized method of moments, proliferation of instruments, principal component analysis, persistence.

\section{Introduction}

Dynamic panel data (DPD) have become very popular in the last two decades, thanks in particular to the increasing availability of panel datasets both at a micro level (e.g. data for individuals, households or firms) and at a macro level (e.g. data for Regions or Countries). The use of dynamic models in macroeconomics dates back to many decades ago, while it is relatively recent in microeconomics. The possibility of including some kind of dynamics also in a microeconomic framework has become very appealing: in fact, it is now a common practice to estimate dynamic models in empirical analysis in most microeconomic fields.

In particular, the generalized method-of-moments (GMM) estimator, in the Holtz-Eakin, Newey and Rosen [1988], Arellano and Bond [1991], Arellano and Bover [1995] and Blundell and Bond [1998] formulations, has gained a leading role among the DPD estimators, mainly due to its flexibility and to the very few assumptions about the data generating process it requires. Most of all, while preventing from the well known DPD bias (see Nickell [1981]) and from the trade off between lag depth and sample size ${ }^{1}$, the GMM estimator also gives the opportunity to account for individual time-invariant effects and for potential endogeneity of regressors. Another advantage is the availability of "internal" instruments (lags of the endogenous variables), a noticeable point when finding instruments is not an easy task. The implementation of ad hoc procedures in many statistical softwares and the consequent availability of "buttons to push" have done the rest of the job.

The GMM estimator however is not the panacea for all the drawbacks of the

\footnotetext{
${ }^{1}$ This former problem is instead an intrinsic and unavoidable characteristic of the AndersonHsiao [1981, 1982] 2SLS estimator for DPD.
} 
previously proposed DPD estimators: it is in fact not free of faults. Instrument proliferation, among the others, is a severe issue in the application of the GMM estimator to DPD models and needs to receive more attention than what it has been done so far. The potential distortions in the estimates by instrumental variables (IV) and GMM estimators when the instrument count gets larger and larger have been treated extensively in the literature ${ }^{2}$, but not enough attention has been paid to this issue in Difference, Level and System GMM estimation of DPD (DIF GMM, LEV GMM and SYS GMM henceforth).

Though these versions of the GMM estimator are designed for a large $\mathrm{N}$ small $T$ framework, and though the time dimension in panel datasets remains well below that of a typical time series, it is well-known that the number of moment conditions increases exponentially with $T$ and the dimension, $m$, of the vector of endogenous regressors other than the lagged dependent variable; this number can get rapidly large relative to the sample size. Consequently, the excessive number of instruments can create a trade-off between bias (overfitting of endogenous variables) and efficiency (additional moment conditions), give an imprecise estimate of the variance/covariance matrix of the moments, lower the power of specification tests (Sargan [1958] / Hansen [1982] test of over-identifying restrictions) and exacerbate the weak instruments problem.

Unfortunately, the problem of instrument proliferation is only rarely detected and addressed in empirical analyses with the consequent risk of drawing misleading conclusions about the coefficient estimates. In many empirical papers, GMM is often applied with unclear specification of the estimator concerning initial weighting matrix, onestep or twostep estimate and, in particular, the selection of instruments: different results emerge as a consequence of different choices of the instrument matrix (for example, how many lags are included) and it becomes difficult to interpret such results as robustness checks, as they are based on a certain degree of arbitrariness, ability or experience of the researcher.

Moreover, there is not a clear indication on how many instruments is too many and on which is a reasonable number of instruments to be used in empirical works.

The paper has two aims. The first one is to introduce a data-driven technique for the reduction of the instrument count in GMM estimation of DPD with other explanatory endogenous variables in addition to the lagged dependent variable. We extract the principal components from the instrument matrix through the principal component analysis (PCA) and use the PCA scores as a new set of instru-

\footnotetext{
²See, among the others, Ziliak [1997] and Bowsher [2002].
} 
ments (we call this procedure principal component-based IV reduction, PCIVR, henceforth). In doing so, we aspire to answer the question "How many moment conditions can be used and still expect to be able to obtain valid inference when estimating by GMM?". Since, in the words of Hall and Peixe [2003, p. 271], "It is impossible to verify a priori which elements of the candidate [instruments] set satisfy [the] conditions [orthogonality, identification, efficiency, and non-redundancy] for a given data set", we suggest a statistically founded rule for the selection of non redundant IVs, based on the characteristics of the empirical problem at hand. In doing so, we extend the analysis of Doran and Schmidt [2006] who consider an eigenvalue-eigenvector decomposition of the variance matrix of the moment conditions, and then discard the terms corresponding to the smallest eigenvalues; they simulate a simple autoregressive DPD and compare results for different autoregressive parameter values, different variance of individual effects, different sample sizes $N$ and $T^{3}$

The second aim of the paper is to fill the gap in the literature by comparing the performance of the Difference, Level and System GMM estimators when various instrument reduction techniques are adopted. In order to do so, we both run extensive Monte Carlo experiments and we estimate economic models on real data, allowing for the presence of endogenous variables (together with the lagged dependent variable), and checking the effects of various persistence characteristics of the stochastic processes, of different sample sizes $N$ and $T$, and of the use of Windmeijer [2005] finite sample correction.

Along with the PCIVR method, the other techniques to reduce the number of moment conditions we compare are the two usually employed in the empirical literature: the collapsing of the instrument matrix (Roodman [2009b]) and the reduction of the lag depth of the instruments. Both solutions make the instrument count linear in $T$ : the former creates different instruments for each lag but not also for each time period; the latter consists of the inclusion as instruments of only few lags instead of all the available ones. Both techniques, separately or combined together, have gained popularity thanks to their direct implementability in the statistical softwares and are now commonly, and often blindly, used in empirical works. ${ }^{4}$ However, collapsing and lag depth truncation involve a certain

\footnotetext{
${ }^{3}$ Mehrhoff [2009] sketches the idea of applying the PCA on the GMM-style instrument matrix in the Difference GMM framework with no additional endogenous regressors and an arbitrary choice of the number of components to be retained.

${ }^{4}$ Other suggestions by the literature had less following in the applied works: the projectionrestricted IV estimation of Arellano [2003] and the canonical correlations and information criteria of Hall and Peixe [2003].
} 
degree of arbitrariness as they ask either to trust the restrictions that are imposed when the instrument matrix is collapsed or to choose how many lags to include among the instruments. Despite some attempts to investigate the performance of the GMM estimators when instrument reduction techniques are employed, the literature in this fields lacks of exhaustive experiments that compare extensively these strategies and their robustness to different settings of the parameters in the simulation model of a DPD with other endogenous variables besides the lagged dependent variable. Our paper aims to fill this gap. ${ }^{5}$

Our results confirm that PCIVR is a general, data-driven technique to reduce overidentification problems that can be fruitfully applied to any overidentified GMM problem. Having tried alternative criteria in order to select the number of retained components (keep only the components whose eigenvalues are larger than the average eigenvalue or retain only the components that explain a given predetermined portion of the original variance), we suggest, as a selection criterion, the explanation of $90 \%$ of the original variance.

In the remainder of the work we proceed as follows: in section 2, after reviewing the collapsing and limiting, we illustrate the extraction of principal components from the instrument matrix and discuss the rationale of applying the PCA on the instrument set; the comparison of a number of instrument reduction techniques is presented by replicating the Blundell and Bond [1998] estimates for the labour demand in the UK and by exploiting extensive Monte Carlo simulations (in section 3); in section 4 we present an empirical application that estimates a production function with three inputs - labour, tangible and intangible capital for a large panel data-set; section 5 draws the conclusions and indicate practical hints for the empirical analysis; the Appendix runs through the technical details of the PCA.

\section{Reducing the instrument count in GMM estimation}

Consider the general one-way error component DPD model:

$$
y_{i t}=\alpha y_{i t-1}+\beta^{\prime} x_{i, t}+\phi_{t}+v_{i t}, v_{i t}=\eta_{i}+\varepsilon_{i t},
$$

where $i=1, . ., N, t=1, . ., T$, $\mathrm{x}$ is a $m$-dimensional vector of potentially endogenous regressors, the $\phi_{t}$ are the time effects (usually considered deterministic), the

\footnotetext{
${ }^{5}$ Roodman [2009b] presents only a Monte Carlo experiment limited to an autoregressive model to compare the collapsing and lag-truncation techniques but restricts the analysis to the System GMM estimator and to a specific parameter setting. Mehrhoff [2009] instead bounds his experiment to the Difference GMM estimator, that is less exposed to instrument proliferation dangers.
} 
$\eta_{i}$ are the individual effects and $\varepsilon_{i t}$ is a zero-mean idiosyncratic error, allowed to be heteroskedastic but not serially correlated. The standard assumptions are: $E\left[\eta_{i}\right]=E\left[\varepsilon_{i t}\right]=E\left[\eta_{i} \varepsilon_{i t}\right]=0$ and predetermined initial conditions $E\left[y_{i 1} \varepsilon_{i t}\right]=0$.

The Arellano-Bond and Arellano-Bover / Blundell-Bond estimators are linear GMM estimators for the model in first differences (DIF GMM) or in levels (LEV GMM) or both (SYS GMM) where the instrument matrix $\mathbf{Z}$ includes the lagged values of the endogenous variables only or also the lagged first differences of the endogenous variables ${ }^{6}$. In the standard framework of DIF and SYS GMM, the columns of the instrument matrix $\mathbf{Z}$ correspond respectively to two different sets of meaningful moment conditions.

In particular, the Arellano-Bond DIF GMM estimator exploits, for each endogenous variable, the following $(T-2)(T-1) / 2$ moment conditions for the equation (1) in first differences: ${ }^{7}$

$$
E\left[\left(\mathbf{Z}_{\mathrm{i}}^{\mathrm{dif}}\right)^{\prime} \Delta v_{i}\right]=E\left[\left(\mathrm{Z}_{\mathrm{it}-l^{\mathrm{dif}}}\right)^{\prime} \Delta v_{i t}\right]=0 \text { for } t \geq 3, l \geq 2
$$

For the sake of simplicity suppose $m=1$; the instrument matrix $\mathbf{Z}^{\text {dif }}$, that satisfies the moment restrictions in (2), contains an IV for each endogenous variable, time period and lag distance and it has the well known form:

$$
Z_{i}^{\text {dif }}=\left(\begin{array}{cccccccccc}
y_{i 1} & x_{i 1} & 0 & \ldots & \ldots & \ldots & \ldots & \ldots & \ldots & 0 \\
\vdots & \vdots & \vdots & \ddots & \vdots & \vdots & \vdots & \ldots & \ldots & 0 \\
0 & 0 & \ldots & 0 & y_{i 1} & \ldots & y_{i T-2} & x_{i 1} & \ldots & x_{i T-2}
\end{array}\right)
$$

The Blundell-Bond SYS GMM estimator also exploits, for each endogenous variable, the additional non-redundant $T-2$ orthogonality conditions for the equation (1) in levels:

$$
E\left[\left(\mathbf{Z}_{\mathrm{i}}^{\text {lev }}\right)^{\prime} v_{i}\right]=E\left[\left(\mathbf{Z}_{\mathrm{is}}{ }^{\text {lev }}\right)^{\prime} v_{i T}\right]=0 \text { for } s=2, \ldots, T-1
$$

\footnotetext{
${ }^{6}$ We use $\mathbf{Z}$ to define a general instrument matrix for DPD GMM estimation. $\mathbf{Z}$ can stand for the untransformed matrix, the collapsed matrix or the limited matrix of instruments. When we need to indicate more precisely the matrix we are considering, we use specific superscripts to denote it.

7 Suitably lagged $x$-variables can also be used as IVs when the $x$-variables are predetermined or strictly exogenous: for predetermined $x$-variables we have $l \geq 1$ and $(T-2)(T+1) / 2$ moment conditions; if they are instead strictly exogenous $l=0$ and the moment conditions are $T(T-2)$.
} 
where, again for $m=1$, the instrument matrix is: ${ }^{8}$

$$
Z_{i}^{\text {lev }}=\left(\begin{array}{ccccc}
\Delta y_{i 2} & \Delta x_{i 2} & 0 & \ldots & 0 \\
\vdots & \vdots & \ddots & \ldots & 0 \\
0 & 0 & \ldots & \Delta y_{i T-1} & \Delta x_{i T-1}
\end{array}\right)
$$

The full instrument matrix for the SYS GMM estimator will thus be:

$$
\mathrm{Z}_{i}^{\text {sys }}=\left(\begin{array}{cc}
\mathrm{Z}_{i}^{\mathrm{dif}} & 0 \\
0 & \mathrm{Z}_{i}^{\text {lev }}
\end{array}\right)
$$

Since usually lags of the explanatory variables are used as IVs, "the phenomenon of moment condition proliferation is far from being a theoretical construct and arises in a natural way in many empirical econometric settings" (Han and Phillips [2006, p. 149]). The dimension of the GMM-type instrument matrix grows exponentially as the number of time periods and regressors expands, even if the time span of the panel is of moderate size.

\subsection{Collapsing and limiting the instrument set}

As discussed in Roodman [2009], when we collapse the instrument set we impose the same condition for all $t$ and we create an instrument for each endogenous variable and lag distance rather than for each endogenous variable, time period and lag distance. The collapsed instrument matrix for the equation in first differences has the form, for $m=1$ :

$$
Z_{i}^{\mathrm{dif}, C}=\left(\begin{array}{cccccc}
y_{i 1} & 0 & x_{i 1} & 0 & 0 & \ldots \\
y_{i 2} & y_{i 1} & x_{i 2} & x_{i 1} & 0 & \ldots \\
\vdots & \vdots & \vdots & \vdots & \vdots & \ddots
\end{array}\right)
$$

with $(T-2)$ moment conditions for each endogenous.

Similarly, the collapsed matrix for the equation in levels is:

$$
Z_{i}^{\text {lev, } C}=\left(\begin{array}{cc}
\Delta y_{i 2} & \Delta x_{i 2} \\
\Delta y_{i 3} & \Delta x_{i 3} \\
\vdots & \vdots
\end{array}\right)
$$

\footnotetext{
${ }^{8}$ The LEV GMM estimation considers, for each endogenous variable, time period and lag distance, all the available lags of the first differences as instrument for the equation in levels because they are non redundant. See Bond [2002] and Bun and Windmeijer [2010] for further discussion on this issue.
} 
The collapsed matrix for the system estimator will thus be:

$$
\mathrm{Z}_{i}^{\text {sys, }} \mathrm{C}=\left(\begin{array}{cc}
\mathrm{Z}_{i}^{\mathrm{dif}, \mathrm{C}} & 0 \\
0 & \mathrm{Z}_{i}^{\mathrm{lev}, \mathrm{C}}
\end{array}\right) .
$$

with $(T-2)+1$ moment conditions for each endogenous variable.

When instead we limit the lag depth, we truncate the moment restrictions and exploit the conditions in equation (2) only for $2 \leq l \leq M$, where $M$ is the maximum lag depth we consider. The limited instrument matrix for the equation in first differences will be:

$$
Z_{i}^{\text {dif, } L}=\left(\begin{array}{ccccccccccccc}
y_{i 1} & x_{i 1} & 0 & 0 & 0 & 0 & 0 & 0 & 0 & 0 & 0 & 0 & \ldots \\
0 & 0 & y_{i 2} & y_{i 1} & x_{i 2} & x_{i 1} & 0 & 0 & 0 & 0 & 0 & 0 & \ldots \\
0 & 0 & 0 & 0 & 0 & 0 & y_{i 3} & y_{i 2} & x_{i 3} & x_{i 2} & 0 & 0 & \ldots \\
\vdots & \vdots & \vdots & \vdots & \vdots & \vdots & \vdots & \vdots & \vdots & \vdots & \vdots & \vdots & \ddots
\end{array}\right)
$$

The number of instruments is $\frac{(T-2)(T-1)}{2}-\frac{(T-2-M)(T-1-M)}{2}$ and the instrument count depends on the number of endogenous variables, on $T$ and on $M$. The truncation in the lag depth has no impact on $Z_{i}^{\text {lev }}$, as it already includes only the first lag available. By limiting arbitrarily the lag depth, we drop from the instrument set $\mathbf{Z}$ all the information about the lags greater than $M$; by collapsing the instrument matrix, we retain a lot more information as none of the lags is actually dropped, though restrictions are imposed on the coefficients of subsets of instruments so that we only generate a single instrument for each lag.

\subsection{Extracting principal components from the matrix of instruments}

In order to face the problem of instrument proliferation, we propose a strategy that involves a stochastic transformation of the instrument set: we extract the principal components from the instrument matrix $\mathbf{Z}$.

The adoption of principal components analysis (PCA) or factor analysis to extract a small number of factors from a large set of variables has become popular in macroeconomic fields of analysis. The main use of factors is in forecasting in second stage regressions, but they are also employed as instrumental variables in IV estimation, in augmented VAR models and in DSGE models ${ }^{9}$. The seminal works by Stock and Watson [1998, 2002a, 2002b] develop the use of static principal components to identify common factors when the number of variables in the

\footnotetext{
${ }^{9}$ Stock and Watson [2010] provide an extensive survey on the use of estimated factors in economic analysis.
} 
dataset gets very large, while Forni et al. [2000, 2004, 2005] propose the use of dynamic principal components. Stock and Watson [2002a] prove consistency of the factors as the number of original variables gets sufficiently large, so that the principal components are estimated precisely enough to be used as data instead of the original variables in subsequent regressions.

The idea of using principal components or factors as instrumental variables is not so new in the literature. Kloek and Mennes [1960] and Amemiya [1966] first proposed the use of principal components in instrumental variable (IV) estimation. In this stream of literature, we find, among the others, important contributions by Kapetanios and Marcellino [2010], Groen and Kapetanios [2009] and by Bai and $\mathrm{Ng}$ [2010] that rely on factor-IV or factor-GMM estimation ${ }^{10}$.

In the stream that uses factors as instruments, the main novelty of what we do here is that we consider a DPD model with endogenous explanatory variables and extract principal components allowing for two strategies: (1) we apply PCA to a large set of lags of each instrument considered separately (what we call PCIV); (2) we apply PCA to a large set of lags of all the different instruments taken together (what we call PCIVT). The idea is that of identifying the most meaningful basis to re-express the information conveyed by the $\mathbf{Z}$, avoiding multicollinearities in the instrument set. This new basis should filter out the noise component of the moment conditions ${ }^{11}$ and reveal the signal delivered by the instrument set (coming from the mean of the sample moment conditions); most important, the noise reduction is the result of a data-driven procedure.

Through the PCA we extract the largest eigenvalues from the estimated covariance $^{12}$ or correlation matrix ${ }^{13}$ of $\mathbf{Z}$ and, by combining the relative eigenvectors, we obtain the loading matrix and the score matrix. We then use the PCA scores as new instrumental variables for the endogenous variables in GMM estimates (PCIVR).

\footnotetext{
${ }^{10} \mathrm{~A}$ review of the literature on Factor-IV and Factor-GMM estimations is in the introduction of Kapetanios and Marcellino [2010].

${ }^{11}$ The degree of variation over the sample moment conditions increases as the number of moment conditions raises

${ }^{12} \mathrm{An}$ unbiased estimator of the covariance matrix of a $p$-dimensional vector $\mathrm{x}$ of random variables is given by the sample covariance matrix $\mathbf{C}=\frac{1}{N-1} \mathbf{X}^{\prime} \mathbf{X}$ where $\mathbf{X}$ is a $N \times p$ zero mean design matrix.

${ }^{13}$ There is not a clear indication in the theoretical literature on which is the preferable matrix among the two. The PCA is scale dependent and the components that are extracted from either matrices are different. The PCA on the covariance matrix can be used when the variables are in commensurable units and have similar variances, as it is generally the case in Monte Carlo experiments. In estimating economic models the PCA on the correlation matrix is instead preferable. We always use PCA on the correlation matrix.
} 
In practice, defined $\mathbf{Z}$ as the general $p$-columns GMM-style instrument matrix ${ }^{14}$, we extract $p$ eigenvalues $\lambda_{1}, \lambda_{2}, \ldots, \lambda_{p} \geq 0$ from the covariance matrix of $\mathbf{Z}$, ordered from the largest to the smallest, and derive the corresponding eigenvectors (principal components) $\alpha_{1}, \alpha_{2}, \ldots, \alpha_{p}$. Our new instruments will be the scores from PCA that are defined as:

$$
\mathbf{s}_{k}=\mathrm{Z} \alpha_{k} \text { for } k=1,2, \ldots, p
$$

If we write $\mathbf{Z}=\left[\begin{array}{llll}\mathbf{z}_{1} & \mathbf{z}_{2} & \ldots & \mathbf{z}_{p}\end{array}\right]$ with $\mathbf{z}_{j}$ being the $j^{\text {th }}$ column of the instrument matrix, the score $s_{k}$ corresponding to the $k^{\text {th }}$ component can therefore be rewritten as:

$$
\mathbf{s}_{k}=\alpha_{k 1} \mathbf{z}_{1}+\alpha_{k 2} \mathbf{z}_{2}+\ldots+\alpha_{k p} \mathbf{z}_{p}
$$

where $\alpha_{k j}$ is the $j^{\text {th }}$ element of the principal component $\alpha_{k}$.

Since the aim of the PCA is data reduction, it would not help to keep all the $p$ scores in the analysis as this would imply no decrease in the number of instruments; only in the first application of section 3 we will check the impact of PCIVR on estimation results when all the $p$ components are retained. In general we suggest to retain only $(m+1) \leq q<p$ principal components; as a consequence, only the $q$ corresponding score vectors will form the new transformed instrument matrix. Alternative criteria can be applied in order to select the components to be retained. ${ }^{15}$ In line with Doran and Schmidt [2006, p. 406], we propose the variability criterion; in particular, we retain the components that explain 90\% of the original variance. With this criterion, the leading eigenvectors from the eigen decomposition of the correlation matrix of the instruments describe a series of uncorrelated linear combinations of the instruments that contain most of the variance. Compared to alternative criteria to select the eigenvalues of interest, we think that retaining principal components that explain a given predetermined portion of the original variance better avoids the magnification of sampling errors in the process of inversion of the variance matrix of the moment conditions. This should decrease the variance of the estimated weighting matrix and improve finite sample performance of the GMM estimator. ${ }^{16}$

\footnotetext{
${ }^{14} \mathbf{Z}$ can be $\mathbf{Z}^{\text {dif }}, \mathbf{Z}^{\text {sys }}, \mathbf{Z}^{\text {dif, }}, \mathbf{Z}^{\text {sys }, C}, \mathbf{Z}^{\text {dif, }}, \mathbf{Z}^{\text {sys, }}$, according to the notation adopted in the previous sections. Remember that, in the simplified case of a balanced panel with $T_{i}=T \forall i$, and $m$ endogenous variables plus the lagged dependent variable, we have: $Z^{\text {dif }}$ has $p=((T-2)(T-$ $1) / 2)(m+1)$ columns, $Z^{\text {dif, } C}$ has $p=(T-2)(m+1)$ columns, $Z^{\text {dif, }, \mathrm{L}}$ a number of columns depending also on the lag truncation. In system GMM estimation, further $(T-2)(m+1)$ columns are added in $\mathbf{Z}^{\text {sys }}$ and in $\mathbf{Z}^{\text {sys, }} \mathbf{L}$, while only $m+1$ are added to $\mathbf{Z}^{\text {sys, },}$.

${ }^{15}$ The criteria are discussed in the Appendix.

${ }^{16}$ According to alternative selection criteria, the smallest eigenvalue or the two or three smallest
} 
Defined the matrix of PCA loadings as $V=\left[\begin{array}{llll}\alpha_{1} & \alpha_{2} & \ldots & \alpha_{p}\end{array}\right]$ and the matrix of PCA scores as $S$, we have that $S=Z V$. Instead of the moment conditions in (2), we will therefore exploit the following restrictions:

$$
E\left[\left(\mathrm{~S}^{\mathrm{dif}}\right)^{\prime} \Delta v\right]=E\left[\left(\mathbf{Z}^{\mathrm{dif}} \mathrm{V}\right)^{\prime} \Delta v\right]=0
$$

Similarly, in the SYS GMM we will also exploit the additional orthogonality conditions

$$
E\left[\left(S^{\operatorname{lev}}\right)^{\prime} v\right]=E\left[\left(Z^{\operatorname{lev} v}\right)^{\prime} v\right]=0
$$

In both cases, the number of moment restrictions depends on the number of components we retain in the analysis that, in turn, depends on the nature of the data at hand. As our starting point is that instruments are orthogonal to the error term, a linear combination of the original instruments will also obviously be orthogonal to the error term.

The rationale of PCIVR is to use, instead of the untransformed instruments, linear combinations of the original instruments that are properly weighed according to the PCA loadings: no available instrument is actually dropped, but its influence might be rescaled after the PCA. It is also worth noticing that none of the instruments that are not in the original matrix $\mathbf{Z}$ will enter the linear combinations which forms the columns of the new instrument matrix. PCA thus preserves all the information in the original instrument set.

A further advantage of PCA is that we can extract principal components not only from the untransformed instrument matrix but also from any transformation we think could be useful; for example, applying PCA to the limited or collapsed instrument matrix would retain all the information each matrix conveys and thus further reduce the number of instruments. As another example, we could apply multistep PCA (see e.g. D'Alessio [1989]) to highlight structural aspects of the data at hand, like persistence or heterogeneity among clusters of individuals.

ones can be arbitrarily dropped; alternatively, one could retain the eigenvalues higher than the average eigenvalue or a fixed number of the highest ones. Results that compare the performance of PCIVR when seveleral of such criteria are applied, as well as under various alternative settings, are available in Mammi [2011]. 


\section{Comparing the instrument reduction techniques}

\subsection{The application of PCIVR technique to Blundell and Bond [1998] model}

In this section we apply our PCIVR technique to the Blundell and Bond [1998] dynamic labour demand equation of p. 135:

$$
n_{i t}=\alpha n_{i t-1}+\beta_{0} w_{i t}+\beta_{1} w_{i t-1}+\gamma_{0} k_{i t}+\gamma_{1} k_{i t-1}+\phi_{t}+\eta_{i}+v_{i t}
$$

where $n_{i t}$ is the $\log$ of employment in firm $i$ in year $t, w_{i t}$ is the $\log$ of the real product wage and $k_{i t}$ is the $\log$ of the capital stock. The sample is an unbalanced panel of 140 UK listed manufacturing companies with between 7 and 9 annual observations over the period 1976-1984. Results are reported in Tables 1, 2 and 3 for DIF, SYS and LEV GMM, respectively; in particular, the first column of Tables 1 and 2 replicate DIF and SYS GMM estimates of the last two columns of Table 4 in Blundell and Bond [1998]. Table 3 adds also LEV GMM estimates. The other columns of Tables 1, 2 and 3 present collapsing (DIFC, SYSc and LEVc), limiting (DIFl, SYSI and LEVI) and PCIVR on each variable separately and on the variables together (DPCIV100, DPCIV90, DPCIVT90; SPCIV100, SPCIV90, SPCIVT90; LPCIV100, LPCIV90, LPCIVT90). Reported estimates are the onestep GMM ones with standard errors robust to heteroskedasticity. The first point to stress is that PCIV100, which uses PCA to just transform the instrument set without dropping any of the moment conditions, does not alter the estimation results originally presented by Blundell and Bond. This is true for each variable of the model, for the specification tests and for different GMM estimates (DIF, SYS or LEV). The retain of the scores that are able to explain $90 \%$ of the original variance (PCIV90) in DIF GMM makes evident the problem of near unit root characterizing the data at hand: lagged wage is no more significant, and Hansen and residuals second-order autocorrelation tests present lower $\mathrm{p}$-values. This a signal of weak instruments due to persistence that specially affect DIF GMM. These problems are exacerbated by PCIVT: putting together all the instruments and their lags, the PCA operates a sort of reduced form between near unit root stochastic processes and therefore, compared to collapse and lag truncation, casts light on the inappropriateness of the instruments. The overfitting of the model with troublesome moment conditions produces a downwards bias of the estimates (in the direction of Within-Group estimates) and a general increase in the variance. Moving to SYS GMM we note that the weak instruments problem due to persistence is reduced, as suggested by Blundell and Bond. Now PCIV90 delivers estimation results that are in line with original SYS GMM more than the 
other instrument reduction techniques, like collapsing and lag truncation. Compared to original SYS GMM, however, the reduced number of moment conditions implied by PCIVR reveals the rejection of the orthogonality conditions through the Hansen test. This rejection can be explained by the use of moment conditions in levels for the equation in differences; when we look at the LEV GMM, in which moment conditions in first differences are used for equations in level, we note how estimation results are close each other and that the Hansen test does not reject the overidentifying restrictions (the persistence of instruments is solved by the first-difference transformation).

\subsection{Monte Carlo experiments: a multivariate dynamic panel data model}

In our set of Monte Carlo simulations we estimate a multivariate DPD whose settings are the same as in Blundell et al. [2000]. The model of interest is:

$$
\begin{aligned}
& y_{i t}=\alpha y_{i t-1}+\beta x_{i t}+\eta_{i}+v_{i t} \\
& x_{i t}=\rho x_{i t-1}+\tau \eta_{i}+\theta v_{i t}+e_{i t}
\end{aligned}
$$

where $\eta_{i} \sim \mathcal{N}\left(0, \sigma_{\eta}^{2}\right)$ are the fixed effects; $v_{i t} \sim \mathcal{N}\left(0, \sigma_{v}^{2}\right)$ and $e_{i t} \sim \mathcal{N}\left(0, \sigma_{e}^{2}\right)$ are the idiosyncratic shocks. Initial observations are drawn from a covariance stationary distribution such that

$$
\begin{gathered}
E\left[\left(x_{i 1}-\frac{\tau \eta_{i}}{1-\rho}\right) \tau \eta_{i}\right]=0 \\
E\left[\left(y_{i 1}-\frac{\beta\left(\frac{\tau \eta_{i}}{1-\rho}\right)+\eta_{i}}{1-\alpha}\right) \eta_{i}\right]=0 .
\end{gathered}
$$

The $x_{i t}$ process is positively correlated with $\eta_{i}$ and the value of $\theta$ is negative to mimic the effects of measurement error. The setting of the parameters in the simulation model is as follows: 


\begin{tabular}{|l|l|}
\hline \hline$\alpha$ & $0.5,0.95$ \\
\hline$\rho$ & $0.5,0.95$ \\
\hline Iterations & 100,1000 \\
\hline $\mathrm{N}$ & 500 \\
\hline $\mathrm{T}$ & $5,8,20$ \\
\hline$\beta$ & 1 \\
\hline$\tau$ & 0.25 \\
\hline$\theta$ & -0.1 \\
\hline$\sigma_{\eta}^{2}$ & 1 \\
\hline$\sigma_{v}^{2}$ & 1 \\
\hline$\sigma_{e}^{2}$ & 0.16 \\
\hline \hline
\end{tabular}

In Tables 4, 5, 6 and 7 we consider 500 individuals and two different time lenghts, i.e. $T=5$, 8; each experiment consists of 1000 iterations; reported estimates are the two-step DIF and SYS GMM estimators, with standard errors robust to heteroskedasticity and with the Windmeijer [2005] finite sample correction. In Tables 8 and 9 we consider 500 individuals and a large temporal span, $T=20$; each experiment consists of 100 iterations and reported estimates are the one-step DIF and SYS GMM estimators, with standard errors robust to heteroskedasticity. We consider different degrees of persistence for $y_{i t}$ and $x_{i t}$, as captured by the autoregressive coefficients $\alpha$ and $\rho$. The displayed results are as follows: mean is the mean of estimates; $p 5$ and $p 95$ are the $5^{\text {th }}$ and the $95^{\text {th }}$ percentiles of estimates; $s d$ is the standard deviation of estimates, Hp mean, min and max are the mean, minimum and maximum of the p-values of the Hansen test and Hdf range is the number of overidentifying restrictions. The main aim of these simulations is to show that the PCIVR statistical approach gives results in line with the most appropriate estimation method, that depends on the parameters' setting and on the temporal length $T$. Compared to collapsing and limiting instruments reduction techniques, PCIVR magnifies the good or bad performance of an estimation method, without altering the core of the results. In the case of stationarity of both variables DIF and SYS GMM provide close results, confirmed by the PCIVR. As the temporal dimension of the sample grows, it becomes more evident the effectiveness of PCIVR in reducing the number of overidentifying restrictions: this is particularly true when all the instruments are considered 
together, as in the case of PCIVT, where the reduction process driven by the characteristics of the simulated data. While collapsing and limiting a priori fix the number of moment conditions, the PCIVR presents a range of overidentifying restrictions which is the wider the larger is $T$.

As we move towards the near unit root case of one or of both variables, the latter scenario being very close to the Blundell and Bond empirical application presented above, SYS GMM provides less biased and more precise estimates. It is particularly remarkable that the collapsing gives the highest standard errors in the case of persistence: this loss in the precision of the estimates is due to non-acceptable constraints on the dynamic structure of the instrument set. PCIVR is generally safer than collapsing and limiting as it provides estimates closer to the true parameters. The only not convincing performance is that of PCIVT in the case of DIF GMM under persistent stochastic processes: in addition to the problems of near unit root in the variables, we have here also an artificial and not economically-grounded correlation structure among the variables that further negatively affects the procedure of principal component extraction. In section 4, we will see that, on the contrary, PCIVT on a set of variables that have an economically-founded relationship has a better and more convincing performance.

\section{An empirical example: old and new panel data methods applied to the controversial issue of production function estimates}

In order to compare the performance of alternative instrument reduction techniques in the estimation of an economic model on real data, we use a production function specification with three inputs - labour, tangible and intangible capital stocks - on a large and unbalanced panel of Italian manufacturing companies over the period 1982-2010. Two main reasons drive our choice. As first motivation, the estimation of production functions from company panel data has become puzzling for panel data estimation methods (e.g. Mairesse and Sassenou [1991], Griliches [1998]). Pooled OLS regressions yield plausible parameter estimates, in line with factor shares and generally consistent with constant return to scale. However these estimates should be biased by omitted heterogeneity and endogeneity issues. Attempts to control for unobserved heterogeneity with within or first-difference transformations tend to yield less satisfactory parameter estimates: "In empirical practice, the application of panel data methods to microdata produced rather unsatisfactory results: low and often insignificant capital 
coefficients and unreasonably low estimates of returns to scale" (Griliches and Mairesse [1998] p. 177; see also the discussion in Mairesse and Hall [1995]). The endogeneity issue arises from the simultaneous choice of output and inputs by the decision maker and from the correlations between firm-effects (efficiency levels of the companies, unknown to the econometrician) and the explanatory variables. It also arises from possible measurement errors in variables: omission of labour and capital intensity-of-utilisation variables - such as hours of work per employees and hours of operation per machine; problems in capital stocks construction (changes in the accounting normative, choice of depreciation rates); lack of distinction between blue and white collars in the labour input; lack of firm-specific prices. Noticeable is the fact that GMM methods are usually applied on first differenced equations using appropriately lagged levels of explanatory variables as instruments, with lag-depth truncation at $\mathrm{t}-3$ (Mairesse and Hall [1996] for France and US; Mairesse and Jaumandreu [2005] for France and Spain; Bontempi and Mairesse [2008] for Italy). The second motivation is that our data-set is a large unbalanced panel with a considerable temporal span and our specification model includes three endogenous explanatory variables. Since the number of available instruments depends on the length of the panel and on the number of endogenous explanatory variables, and it changes from cross-section to cross-section, the GMM estimation procedures become very complex, calling for a fruitful use of PCIVR techniques in reducing overfitting problems. Table 10 shows the by-year and by-industry sample composition. Data are drawn from the CADS (Company Accounts Data Service of Centrale dei Bilanci), which is highly representative of the population of Italian companies, covering over 50\% of the value-added produced by those companies included in the Italian Central Statistical Office's Census (further details, cleaning rules and definitions of variables are in Bontempi and Mairesse [2008]). The total number of observations, more than 717,000, is roughly equally splitted between services and manufacturing companies; the total number of individuals is 73,072 , with the availability of minimum 4 years and of maximum 29 years. In order to produce estimation results in line with those of the literature on production function estimates and to preserve the handiness of the empirical framework, we proceed with only the manufacturing companies. We also split the temporal span in two periods, 19821993 and 1995-2010, so that we can check the robustness of our findings to changes in the macroeconomic context. ${ }^{17}$

\footnotetext{
${ }^{17}$ It is worthy to be noted the change of the accounting standards - particularly for the capital stock - following the implementation of the Fourth European Commission Directive since 1993.
} 
The standard model proposed by the literature is the Cobb-Douglas production function with multiplicative specification of the total capital and constant (but non-unity) elasticity of substitution:

$$
Q_{i t}=A_{i} B_{t} L_{i t}^{\beta} C_{i t}^{\alpha} K_{i t}^{\gamma} e_{i t}^{\varepsilon}
$$

where $Q$ indicates the value added; the terms $A_{i}$ and $B_{t}$ respectively capture efficiency (unmeasurable firm-specific characteristics, like management ability) and the state of technology (the macroeconomic events that affect all companies, like business cycle and "disembodied technical changes" i.e. changes over time in the rates of productivity growth); labels $C, K$ and $L$ are tangible and intangible capital stocks and labour, respectively, with the associated parameters measuring the output elasticity to each input; $\varepsilon_{i t}$ is the usual idiosyncratic shocks, allowed to be heteroskedastic and within-firm autocorrelated. ${ }^{18}$

By taking the logarithms of equation 19, and defining all the variables per employee, the multiplicative production function specification becomes:

$$
\left(q_{i t}-l_{i t}\right)=a_{i}+b_{t}+(\mu-1) l_{i t}+\alpha\left(c_{i t}-l_{i t}\right)+\gamma\left(k_{i t}-l_{i t}\right)+\varepsilon_{i t}
$$

where lower-case letters denote logarithms; $a_{i}$ and $b_{t}$ are the usual individual and time effects. Table 11 reports, over the columns, the main statistics of the variables in model 20. In line with the Italian manufacturing division, the data-set is mainly characterized by small and medium-sized firms (with a median number of employees equal to 46 units; about 113 units on average). ${ }^{19}$ Input variables are characterized by outliers causing departures of non-parametric measures of spread (inter-quartile range, iqr) from parametric ones (standard deviation, sd). This is particularly evident in intangible capital stock, suggesting that large intangible stocks are concentrated in relatively few companies, and that zeros more prevail here than in the other two inputs. The decomposition of standard deviation in its between, within and residual components shows that the across companies variability prevails, with shares higher than $60 \%$ (in line with the findings in Griliches [1988]). Table 12 presents correlations among the variables of equation 20 and tangible and intangible gross investments (inv and iinv, respectively); we shall return to this point below, in discussing the role of "internal" (lags of endogenous explanatory variables) and "external" (variables not included in the

\footnotetext{
${ }^{18}$ Note that we assume a one-period gestation lag before intangible and tangible stocks become fully productive; beginning-of-period capital measures avoid the simultaneous correlation between capital inputs and the disturbance term.

${ }^{19}$ The average Italian limited liability company employs 44 workers.
} 
equation of interest but suggested by the economic structure of the problem at hand) instruments in GMM applications. For now, we note that investments are highly correlated with the endogenous variables of equation 20.

Table 13 presents estimation results for the sub-period 1982-1993. The first three columns report, as benchmarks, pooled OLS estimates (biased by the omission of firm-specific effects, correlated with explanatory variables), and within and first-differences estimates, both accounting for cross-sectional heterogeneity. The first-differences estimates are affected by random year-by-year noise that hides the signal of data (Griliches and Hausman [1986]); its effect is particularly evident in the elasticity of labour, and produces disappointing decreasing returns to scale. The following five columns of Table 13 compare DIF GMM estimates with usual "internal" instruments: it is noticeable the lack of robustness in estimation results accordingly to the different technique used to reduce the number of moment conditions and the rejection of overidentifying restrictions by the Hansen test; PCIVR and PCIVRT produce the best results. Estimates further improve as we move towards the last five columns of the Table, in which "external" instruments are used: particularly in the case of PCIVRT, overidentifying restrictions are not rejected and, at least, elasticities of the output to the capital stocks go in the direction of more sensible results. We prefer the "external" instrument to the "internal" ones, for at least one reason: the lags of the explanatory variables may be affected by the same measurement error (possibly correlated over time) that we are trying to tackle. In general, however, the difficulty with DIF GMM estimates is that the past levels of variables are poor instruments for the current differences of the explanatory variables; this even in a large cross-sectional dimension, as in our case, see Bound et al. [1995]. Under covariance stationarity assumptions of the variables in equation 20 we use past differences of investment as ("external") instruments for the levels of productive inputs; accordingly to the above cited literature, LEV GMM - more than DIF GMM - keeps the relevant information in the variables of interest. Results are presented in Table 14 for the two 1982-1993 and 1995-2010 sub-periods. The estimates are encouraging, because robust to changes in the sample periods and in the temporal span, with a non-rejection by the Hansen test that is more evident in the most recent period; moreover, previous disappointing decreasing returns to scale have vanished in favour of constant returns to scale (from an economic point of view, in the first period, or both in economic and statistical terms in the second period) ${ }^{20}$. It is

\footnotetext{
${ }^{20}$ These estimates of elasticities of output with respect to inputs are consistent with evidence for other countries obtained by using constrained models - like the total factor productivity approach - to avoid endogeneity and GMM estimating problems
} 
also remarkable the good performance of PCIVRT in an economic context in which the reduced form behind the production function contemplates the possibility of complementarities among productive inputs (which are magnified by the principal components extraction when the instruments and their lags are putted together). Compared to PCIVR, collapsing and lag truncation present worse results: estimated elasticities for some inputs are less in line with not-reduced GMM and PCIVR, and present lower precision. The not-convincing result obtained with lag-depth truncation of the instrument set should be paid a particular attention, as this reduction strategy is commonly adopted in the literature on productivity.

\section{Conclusions}

This paper introduces a new strategy to reduce the number of instruments in the GMM estimation of dynamic panel data, namely the extraction of principal components from the instrument matrix (PCIVR), and compares the alternative instrument reduction techniques through Monte Carlo simulations and empirical applications.

First, we discussed the rationale of applying the PCA on the instrument matrix stressing that it involves a purely data-driven procedure which does not require particular assumptions on the coefficient of the matrix: it is instead the most information-preserving technique among those we discuss here.

Secondly, we both use empirical applications and run extensive Monte Carlo simulations of multivariate DPD model with endogenous variables additional to the lagged dependent one. We found that the extraction of principal components from the instrument matrix tends to improve GMM results when the assumptions under DIF or LEVISYS GMM are valid.

In the light of the previous findings, we are able to suggest some indications for applied research and to sketch some potential extensions of this work.

Overall, the extraction of principal components from the instrument set seems to be a promising approach to the issue of instrument proliferation: in fact it appears reasonable to exploit the correlations between the instruments to summarize the original information. Our results confirm that PCIVR is a general, data-driven technique to reduce overidentification problems that can be fruitfully applied to any overidentified GMM problem. We suggest the researcher on always reporting the number of instruments and not to adopt an instrument reduction technique a priori, as every strategy could have serious drawbacks if some assumptions do not hold. 
Proper procedures to extract principal components from the instrument matrix have been programmed by the authors in the software Stata: these are based on the preliminary construction of the instrument matrices. This availability could facilitate the researchers in presenting the estimates obtained with alternative GMM estimators with and without data-driven instrument reduction techniques.

Further developments can go in the direction of merging our PCIVR with statistical tests on the validity of the moment conditions. The reduction in the number of overidentifying restrictions should improve the reliability of tests on instruments' validity. In particular, we are going in the direction of multi-step principal components analysis, which involves the identification of "reference" matrices of instruments that enlighten aspects of the data at hand that are problematic for the validity of the instruments; among these, the characteristics of persistence of the instruments. 
Table 1: BB98 model: comparison between GMM DIF estimates

\begin{tabular}{|c|c|c|c|c|c|c|c|}
\hline \multicolumn{2}{|c|}{ Variable } & \multirow{2}{*}{$\frac{\text { DIF }}{0.707}$} & \multirow{2}{*}{$\frac{\text { DIFc }}{0.840}$} & \multirow{2}{*}{$\frac{\text { DIFl }}{0.787}$} & \multirow{2}{*}{$\begin{array}{r}\text { DPCIV100 } \\
0.707\end{array}$} & \multirow{2}{*}{$\begin{array}{r}\text { DPCIV90 } \\
0.802\end{array}$} & \multirow{2}{*}{$\begin{array}{r}\text { DPCIVT90 } \\
0.508\end{array}$} \\
\hline$n$ & coeff & & & & & & \\
\hline & se & 0.084 & 0.107 & 0.120 & 0.084 & 0.126 & 0.179 \\
\hline & $p$ & 0.000 & 0.000 & 0.000 & 0.000 & 0.000 & 0.005 \\
\hline \multirow[t]{3}{*}{$w$} & coeff & -0.709 & -0.971 & -0.662 & -0.709 & -0.862 & -0.675 \\
\hline & se & 0.117 & 0.290 & 0.193 & 0.117 & 0.210 & 0.269 \\
\hline & $p$ & 0.000 & 0.001 & 0.001 & 0.000 & 0.000 & 0.012 \\
\hline \multirow[t]{3}{*}{$w_{t-1}$} & coeff & 0.500 & 0.632 & 0.617 & 0.500 & 0.222 & 0.315 \\
\hline & se & 0.111 & 0.163 & 0.130 & 0.111 & 0.294 & 0.235 \\
\hline & p & 0.000 & 0.000 & 0.000 & 0.000 & 0.450 & 0.179 \\
\hline \multirow[t]{3}{*}{$k$} & coeff & 0.466 & 0.632 & 0.479 & 0.466 & 0.578 & 0.654 \\
\hline & se & 0.101 & 0.215 & 0.139 & 0.101 & 0.225 & 0.209 \\
\hline & $p$ & 0.000 & 0.003 & 0.001 & 0.000 & 0.010 & 0.002 \\
\hline \multirow[t]{3}{*}{$k_{t-1}$} & coeff & -0.215 & -0.547 & -0.438 & -0.215 & -0.411 & -0.200 \\
\hline & se & 0.086 & 0.192 & 0.111 & 0.086 & 0.195 & 0.236 \\
\hline & $p$ & 0.012 & 0.004 & 0.000 & 0.012 & 0.035 & 0.397 \\
\hline \multicolumn{2}{|c|}{ Hansen } & 88.797 & 14.622 & 35.693 & 88.797 & 23.432 & 17.197 \\
\hline \multicolumn{2}{|c|}{ Hansenp } & 0.211 & 0.553 & 0.389 & 0.211 & 0.136 & 0.102 \\
\hline \multicolumn{2}{|c|}{ Hansen $d f$} & 79 & 16 & 34 & 79 & 17 & 11 \\
\hline \multicolumn{2}{|l|}{$\operatorname{ar} 1 p$} & 0.000 & 0.000 & 0.000 & 0.000 & 0.001 & 0.055 \\
\hline \multicolumn{2}{|l|}{$\operatorname{ar} 2 p$} & 0.891 & 0.901 & 0.929 & 0.891 & 0.544 & 0.547 \\
\hline \multicolumn{2}{|l|}{ Obs. } & 751 & 751 & 751 & 751 & 751 & 751 \\
\hline
\end{tabular}


Table 2: BB98 model: comparison between GMM SYS estimates

\begin{tabular}{|c|c|c|c|c|c|c|c|}
\hline \multicolumn{2}{|c|}{ Variable } & \multirow{2}{*}{$\frac{\text { SYS }}{0.811}$} & \multirow{2}{*}{$\frac{\text { SYSc }}{0.777}$} & \multirow{2}{*}{$\frac{\text { SYSI }}{0.841}$} & \multirow{2}{*}{$\begin{array}{r}\text { SPCIV100 } \\
0.809\end{array}$} & \multirow{2}{*}{$\begin{array}{r}\text { SPCIV90 } \\
0.902\end{array}$} & \multirow{2}{*}{$\begin{array}{r}\text { SPCIVT90 } \\
0.857\end{array}$} \\
\hline$n$ & coeff & & & & & & \\
\hline & se & 0.058 & 0.068 & 0.059 & 0.058 & 0.048 & 0.068 \\
\hline & $p$ & 0.000 & 0.000 & 0.000 & 0.000 & 0.000 & 0.000 \\
\hline \multirow[t]{3}{*}{$w$} & coeff & -0.795 & -0.875 & -0.784 & -0.796 & -0.742 & -0.724 \\
\hline & se & 0.097 & 0.260 & 0.148 & 0.097 & 0.154 & 0.150 \\
\hline & $p$ & 0.000 & 0.001 & 0.000 & 0.000 & 0.000 & 0.000 \\
\hline \multirow[t]{3}{*}{$w_{t-1}$} & coeff & 0.550 & 0.693 & 0.560 & 0.547 & 0.464 & 0.560 \\
\hline & se & 0.152 & 0.255 & 0.179 & 0.153 & 0.195 & 0.180 \\
\hline & $p$ & 0.000 & 0.007 & 0.002 & 0.000 & 0.017 & 0.002 \\
\hline \multirow[t]{3}{*}{$k$} & coeff & 0.429 & 0.604 & 0.506 & 0.429 & 0.534 & 0.540 \\
\hline & se & 0.076 & 0.210 & 0.078 & 0.076 & 0.096 & 0.098 \\
\hline & $p$ & 0.000 & 0.004 & 0.000 & 0.000 & 0.000 & 0.000 \\
\hline \multirow[t]{3}{*}{$k_{t-1}$} & coeff & -0.280 & -0.434 & -0.380 & -0.280 & -0.441 & -0.414 \\
\hline & se & 0.078 & 0.246 & 0.079 & 0.078 & 0.103 & 0.097 \\
\hline & $p$ & 0.000 & 0.078 & 0.000 & 0.000 & 0.000 & 0.000 \\
\hline \multicolumn{2}{|c|}{ Hansen } & 115.726 & 17.997 & 70.504 & 115.347 & 57.597 & 42.518 \\
\hline \multicolumn{2}{|c|}{ Hansenp } & 0.135 & 0.523 & 0.078 & 0.140 & 0.022 & 0.022 \\
\hline \multicolumn{2}{|c|}{ Hansen $d f$} & 100 & 19 & 55 & 100 & 38 & 26 \\
\hline \multicolumn{2}{|l|}{$\operatorname{ar} 1 p$} & 0.000 & 0.000 & 0.000 & 0.000 & 0.000 & 0.000 \\
\hline \multicolumn{2}{|l|}{$a r 2 p$} & 0.934 & 0.975 & 0.920 & 0.931 & 0.785 & 0.905 \\
\hline \multicolumn{2}{|l|}{ Obs. } & 891 & 891 & 891 & 891 & 891 & 891 \\
\hline
\end{tabular}


Table 3: BB98 model: comparison between GMM LEV estimates

\begin{tabular}{|c|c|c|c|c|c|c|c|}
\hline \multicolumn{2}{|c|}{ Variable } & LEV & LEVc & LEVI & LPCIV100 & LPCIV90 & LPCIVT90 \\
\hline \multirow[t]{3}{*}{$n$} & coeff & 0.944 & 0.893 & 0.934 & 0.944 & 0.944 & 0.927 \\
\hline & se & 0.022 & 0.091 & 0.033 & 0.022 & 0.027 & 0.026 \\
\hline & $p$ & 0.000 & 0.000 & 0.000 & 0.000 & 0.000 & 0.000 \\
\hline \multirow[t]{3}{*}{$w$} & coeff & -0.606 & -0.730 & -0.809 & -0.606 & -0.776 & -0.723 \\
\hline & se & 0.167 & 0.239 & 0.166 & 0.167 & 0.162 & 0.158 \\
\hline & $p$ & 0.000 & 0.002 & 0.000 & 0.000 & 0.000 & 0.000 \\
\hline \multirow[t]{3}{*}{$w_{t-1}$} & coeff & 0.500 & 0.725 & 0.552 & 0.500 & 0.609 & 0.612 \\
\hline & se & 0.177 & 0.221 & 0.175 & 0.177 & 0.159 & 0.164 \\
\hline & $p$ & 0.005 & 0.001 & 0.002 & 0.005 & 0.000 & 0.000 \\
\hline \multirow[t]{3}{*}{ k } & coeff & 0.522 & 0.831 & 0.500 & 0.522 & 0.516 & 0.565 \\
\hline & se & 0.062 & 0.126 & 0.068 & 0.062 & 0.065 & 0.060 \\
\hline & $p$ & 0.000 & 0.000 & 0.000 & 0.000 & 0.000 & 0.000 \\
\hline \multirow[t]{3}{*}{$k_{t-1}$} & coeff & -0.477 & -0.763 & -0.444 & -0.477 & -0.468 & -0.510 \\
\hline & se & 0.068 & 0.161 & 0.074 & 0.068 & 0.070 & 0.066 \\
\hline & $p$ & 0.000 & 0.000 & 0.000 & 0.000 & 0.000 & 0.000 \\
\hline \multicolumn{2}{|c|}{ Hansen } & 86.805 & 17.657 & 49.700 & 86.805 & 62.608 & 60.454 \\
\hline \multicolumn{2}{|c|}{ Hansenp } & 0.257 & 0.344 & 0.040 & 0.257 & 0.455 & 0.148 \\
\hline \multicolumn{2}{|c|}{ Hansen $d f$} & 79 & 16 & 34 & 79 & 62 & 50 \\
\hline \multicolumn{2}{|l|}{ ar1p } & 0.135 & 0.380 & 0.037 & 0.135 & 0.018 & 0.092 \\
\hline \multicolumn{2}{|l|}{$a r 2 p$} & 0.912 & 0.543 & 0.080 & 0.912 & 0.232 & 0.487 \\
\hline \multicolumn{2}{|l|}{ Obs. } & 891 & 891 & 891 & 891 & 891 & 891 \\
\hline
\end{tabular}




\begin{tabular}{|c|c|c|c|c|c|c|c|c|}
\hline 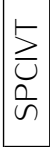 & 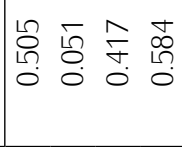 & 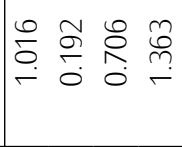 & 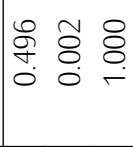 & $\begin{array}{l}a \\
\infty \\
\infty\end{array}$ & 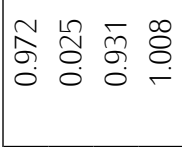 & 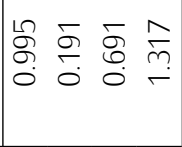 & $\left|\begin{array}{ccc}0 & 8 & 8 \\
\multirow{2}{*}{} & 0 & 0 \\
0 & 0 & 0\end{array}\right|$ & $\infty$ \\
\hline 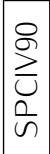 & 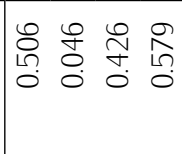 & 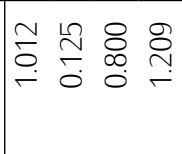 & 章 & $\circ$ & 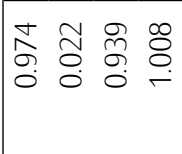 & 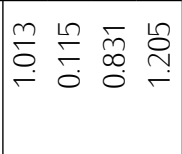 & $\left|\begin{array}{ccc}\bar{f} & 0 & 8 \\
\hdashline & 0 & 0 \\
\circ & 0 & -\end{array}\right|$ & 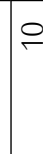 \\
\hline$\frac{\vec{n}}{n}$ & 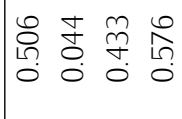 & 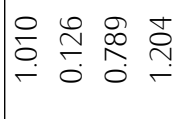 & \begin{tabular}{lll}
$\bar{\sigma}$ & $\bar{\sigma}$ & 8 \\
\hdashline & 8 \\
0 & 0 & 0
\end{tabular} & $\stackrel{+}{+}$ & 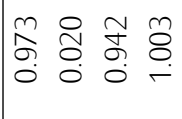 & 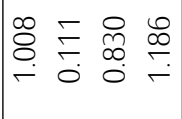 & 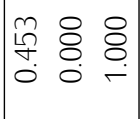 & 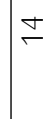 \\
\hline $\begin{array}{l}u \\
\tilde{n} \\
\omega\end{array}$ & 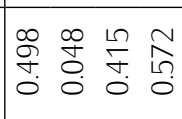 & 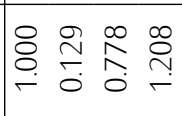 & $\begin{array}{lll}0 & m & \infty \\
0 & 0 & 0 \\
0 & 0 & 0 \\
0 & 0 & 0\end{array}$ & 0 & 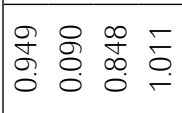 & 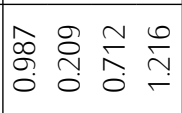 & $\left|\begin{array}{lll}\bar{\delta} & \bar{\sigma} & 0 \\
i & 8 \\
0 & 0 & 0 \\
0\end{array}\right|$ & 0 \\
\hline$\frac{n}{n}$ & 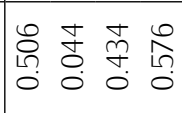 & 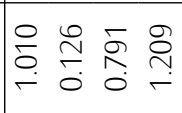 & 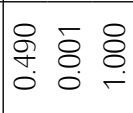 & 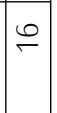 & 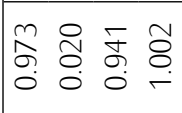 & 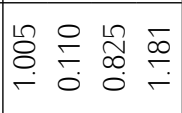 & 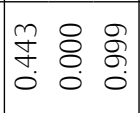 & $\stackrel{0}{\circ}$ \\
\hline $\mid \begin{array}{l}5 \\
\vdots \\
0 \\
0 \\
0\end{array}$ & 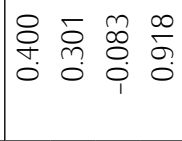 & 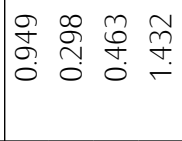 & \begin{tabular}{lll}
$\bar{g}$ & $\bar{\sigma}$ & $\infty$ \\
\hdashline & 0 \\
0 & 0 \\
0 & 0
\end{tabular} & $\stackrel{+}{m}$ & 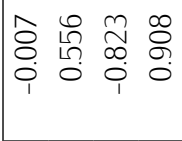 & 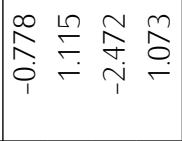 & $\left|\begin{array}{lll}+ & \overline{8} & 8 \\
& 8 & 0 \\
0 & 0 & -\end{array}\right|$ & m \\
\hline $\mid \begin{array}{l}8 \\
2 \\
\vdots \\
0 \\
0\end{array}$ & 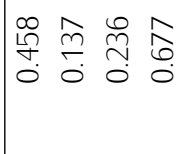 & 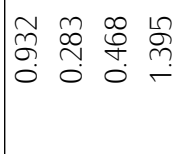 & \begin{tabular}{lll}
$\bar{\sigma}$ & $\bar{\sigma}$ & $\infty$ \\
\hdashline & 0 \\
0 & 0 \\
0 & 0
\end{tabular} & t & 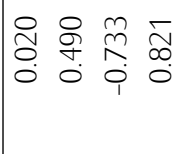 & 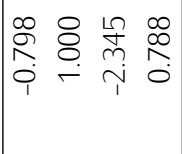 & $\mid$\begin{tabular}{lll}
$\infty$ & 8 & 8 \\
\hdashline & 0 & 0 \\
\hdashline & 0 & 0 \\
\hdashline & 0
\end{tabular} & • \\
\hline$\overline{\bar{\sigma}}$ & 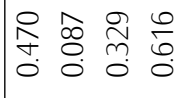 & ‡r & 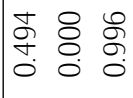 & $\infty$ & 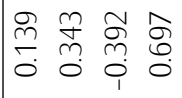 & 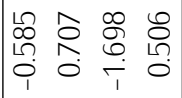 & $\left|\begin{array}{ccc}\bar{r} & 8 & \infty \\
\hdashline & 0 & 0 \\
& 0 & 0 \\
0\end{array}\right|$ & $\infty$ \\
\hline 嵩 & 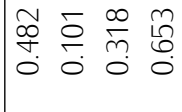 & 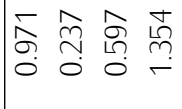 & 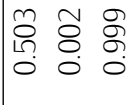 & t & 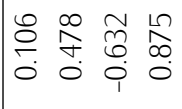 & 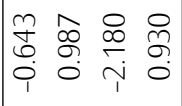 & $\left|\begin{array}{ccc}8 & 8 & 8 \\
\hdashline & 0 & 0 \\
\circ & 0 & 0 \\
-\end{array}\right|$ & • \\
\hline 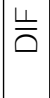 & 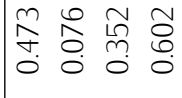 & 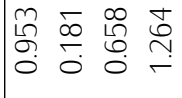 & \begin{tabular}{lll}
0 & $\overline{0}$ \\
\hdashline & $\overline{8}$ & 0 \\
0 & 0 & 0 \\
0 & 0 & 0
\end{tabular} & 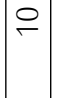 & 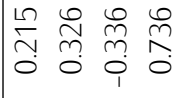 & 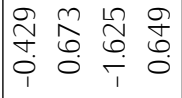 & 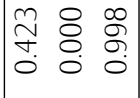 & $\stackrel{0}{\circ}$ \\
\hline & 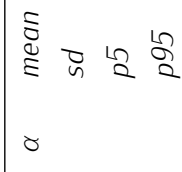 & 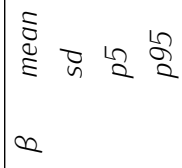 & 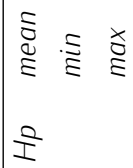 & 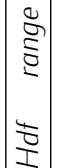 & 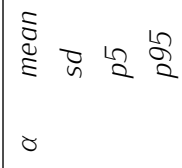 & 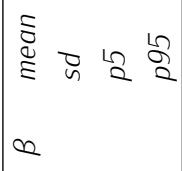 & 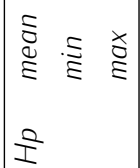 & $\begin{array}{l}8 \\
\vdots \\
\vdots \\
\vdots \\
\vdots \\
\text { I }\end{array}$ \\
\hline & & & & & & $\begin{array}{l}\text { Ln } \\
0 \\
0 \\
0 \\
0\end{array}$ & & \\
\hline
\end{tabular}




\begin{tabular}{|c|c|c|c|c|c|c|c|c|}
\hline $\begin{array}{l}i \\
u \\
\vdots \\
n\end{array}$ & 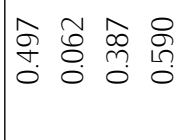 & 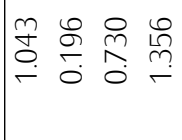 & $\left|\begin{array}{lll}\overline{0} & \overline{8} & 8 \\
\hdashline & 8 & 0 \\
0 & 0 & -\end{array}\right|$ & $\hat{b}$ & 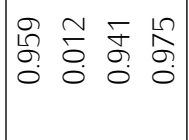 & 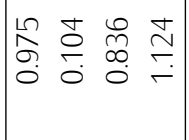 & 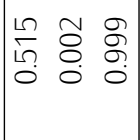 & $\hat{b}$ \\
\hline 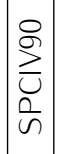 & 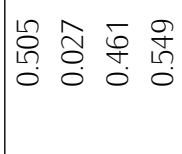 & 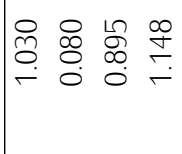 & $\left|\begin{array}{lll}0 & 8 & 8 \\
7 & 8 & 8 \\
0 & 0 & \ddots\end{array}\right|$ & 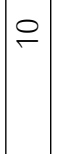 & 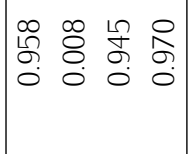 & 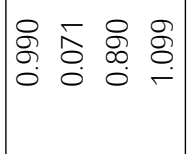 & $\mid \begin{array}{lll}\bar{\tau} & 8 & 8 \\
i n & 8 & 8 \\
0 & 0 & \circ\end{array}$ & 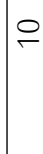 \\
\hline $\begin{array}{l}\bar{n} \\
\frac{\pi}{n}\end{array}$ & 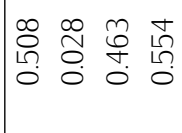 & 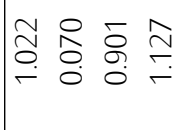 & $\left|\begin{array}{lll}0 & 8 & 8 \\
\dddot{m} & 8 & 8 \\
0 & 0 & -\end{array}\right|$ & 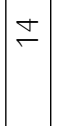 & $\left|\begin{array}{llll}\infty & 1 & 0 & 0 \\
: & 8 & 0 & 0 \\
0 & 0 & 0 \\
0 & 0 & 0 & 0\end{array}\right|$ & 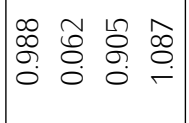 & $\left|\begin{array}{lll}\tilde{0} & \overline{8} & 8 \\
1 & 8 & 0 \\
0 & 0 & -\end{array}\right|$ & $\stackrel{+}{\sim}$ \\
\hline $\begin{array}{c}u \\
\tilde{n} \\
n\end{array}$ & 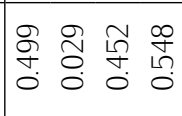 & 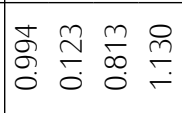 & 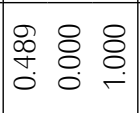 & 0 & 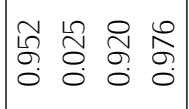 & 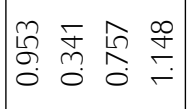 & $\mid \begin{array}{ccc} & 0 & 8 \\
i n & 0 & 0 \\
0 & 0 & 0\end{array}$ & 0 \\
\hline$\frac{n}{n}$ & 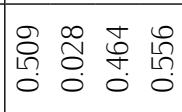 & 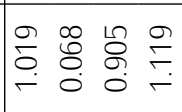 & 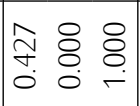 & 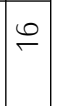 & 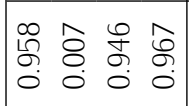 & 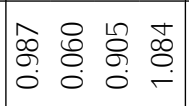 & $\mid \begin{array}{lll}8 & 0 & 8 \\
\mathscr{g} & 8 & 8 \\
0 & 0 & 0 \\
\end{array}$ & $\stackrel{0}{\circ}$ \\
\hline $\mid \begin{array}{l}1 \\
\vdots \\
0 \\
0 \\
0\end{array}$ & 남 & 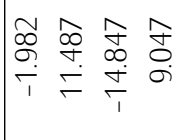 & $\left|\begin{array}{lll}\infty & 8 & 8 \\
\infty & 8 & 8 \\
\hdashline & 0 & \circ \\
0 & 0 & 0\end{array}\right|$ & - & 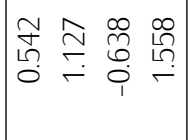 & 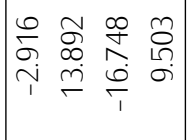 & $\mid \begin{array}{lll}0 & 8 & 8 \\
0 & 8 & 8 \\
0 & 0 & 0 \\
0 & 0 & -\end{array}$ & - \\
\hline $\mid \begin{array}{l}8 \\
9 \\
2 \\
0 \\
0 \\
0\end{array}$ & 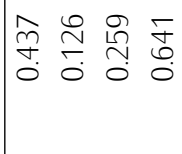 & 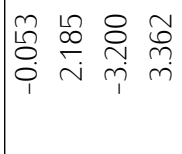 & $\left|\begin{array}{lll}1 & 5 & 8 \\
1 & 8 & 8 \\
0 & 0 & -\end{array}\right|$ & $\nabla$ & 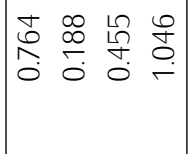 & 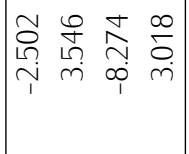 & $\left|\begin{array}{lll}\hat{\beta} & \bar{\sigma} & 8 \\
i & 8 \\
0 & 0 & 0 \\
0\end{array}\right|$ & + \\
\hline$\overline{\bar{\sigma}}$ & 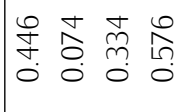 & 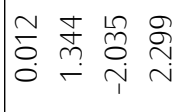 & $\left|\begin{array}{lll}8 & 8 & \infty \\
\hdashline & 8 & 9 \\
\hdashline & 0 & 0 \\
0 & 0 & 0\end{array}\right|$ & $\infty$ & 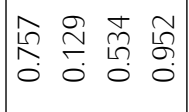 & 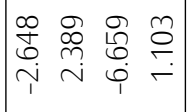 & 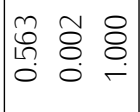 & $\infty$ \\
\hline 訔 & $\mid$\begin{tabular}{llll}
$\infty$ & $\infty$ & 0 & $\infty$ \\
\multirow{f}{*}{} & 0 & 0 & 0 \\
0 & 0 & 0 & 0 \\
0
\end{tabular} & 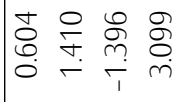 & 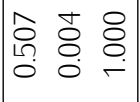 & + & 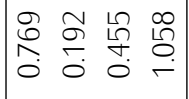 & 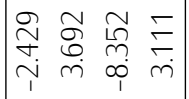 & 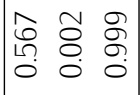 & + \\
\hline$\stackrel{ }{\overline{0}}$ & 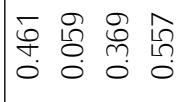 & 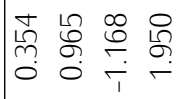 & 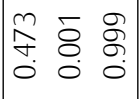 & $\circ$ & 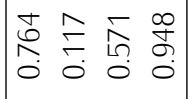 & 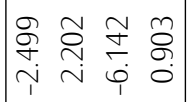 & $\mid \begin{array}{lll}\infty & \bar{\sigma} & 8 \\
& 8 & 0 \\
\circ & 0 & 0 \\
-\end{array}$ & $\stackrel{?}{\circ}$ \\
\hline & 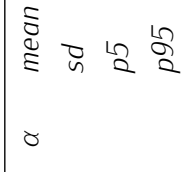 & 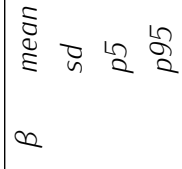 & 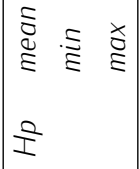 & 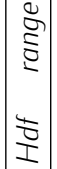 & 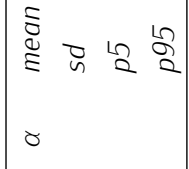 & 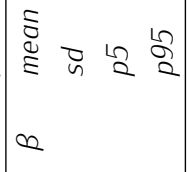 & 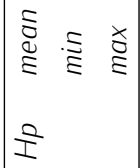 & 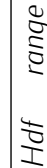 \\
\hline & & & & & & $\begin{array}{l}0 \\
0 \\
0 \\
0\end{array}$ & & \\
\hline
\end{tabular}




\begin{tabular}{|c|c|c|c|c|c|c|c|c|}
\hline $\begin{array}{l}\sum_{\geq} \\
\vdots \\
n\end{array}$ & 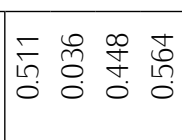 & 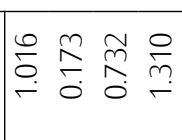 & $\left|\begin{array}{lll}\infty & \overline{8} & 8 \\
\stackrel{\infty}{*} & 8 & 0 \\
0 & 0 & \ddots\end{array}\right|$ & 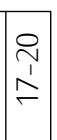 & 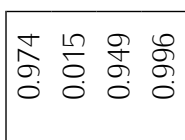 & 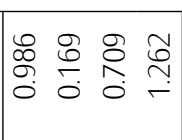 & 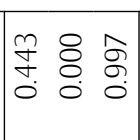 & $\begin{array}{l}\infty \\
1 \\
1 \\
1\end{array}$ \\
\hline $\begin{array}{l}\frac{8}{g} \\
\geq \\
\frac{1}{n} \\
\end{array}$ & 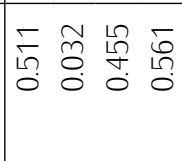 & 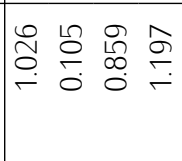 & 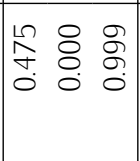 & 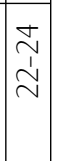 & 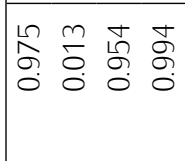 & 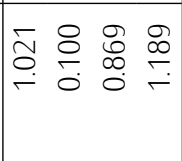 & 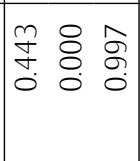 & $\stackrel{\approx}{\approx}$ \\
\hline$\frac{\vec{n}}{\tilde{\omega}}$ & 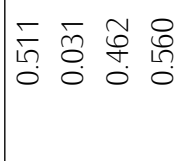 & 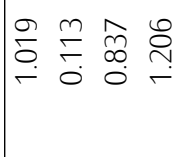 & 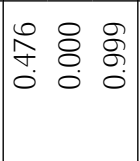 & m & 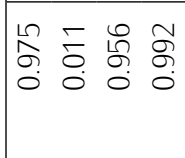 & 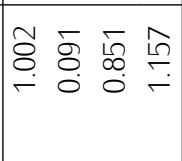 & 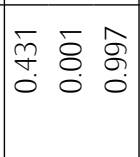 & $\approx$ \\
\hline 崩 & 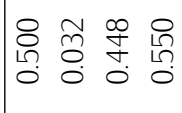 & 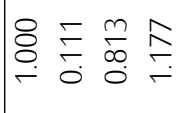 & $\left|\begin{array}{lll}0 & 0 & \infty \\
\multirow{f}{f}{} & 8 & \stackrel{9}{2} \\
0 & 0 & 0 \\
0 & 0 & 0\end{array}\right|$ & $\approx$ & 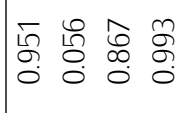 & 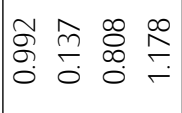 & 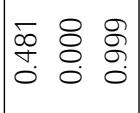 & $\approx$ \\
\hline 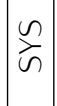 & 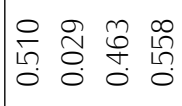 & 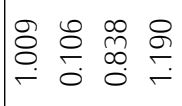 & $\left|\begin{array}{lll}\frac{1}{f} & \bar{\sigma} & 0 \\
0 & 0 & 0 \\
0 & 0 & 0\end{array}\right|$ & กิ & 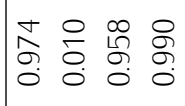 & 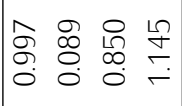 & $\left|\begin{array}{lll}\infty & 0 & \vdots \\
0 & 0 & 0 \\
0 & 0 & 0 \\
0\end{array}\right|$ & in \\
\hline $\mid \begin{array}{l}1 \\
\geq \\
0 \\
0\end{array}$ & 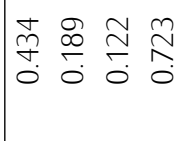 & 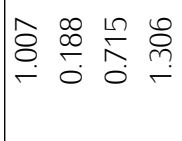 & 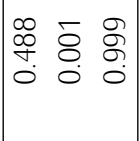 & $\stackrel{9}{i}$ & 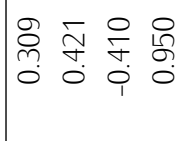 & 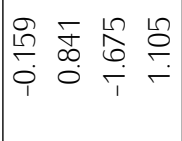 & $\left|\begin{array}{lll}0 & 8 & 9 \\
0 & 8 & 9 \\
0 & 8 & \sigma \\
0 & 0 & 0\end{array}\right|$ & o \\
\hline 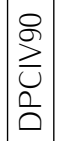 & 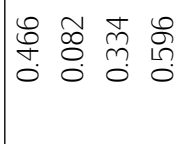 & 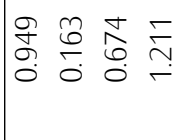 & $\left|\begin{array}{lll}8 & 8 & 8 \\
\frac{9}{+} & 8 & 8 \\
0 & 0 & -\end{array}\right|$ & 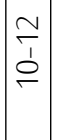 & 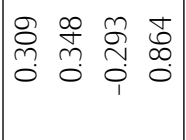 & 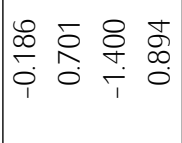 & 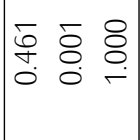 & $\bar{z}$ \\
\hline$\overline{\bar{D}}$ & 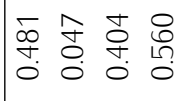 & 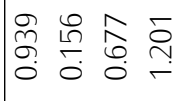 & $\left|\begin{array}{ccc}0 & 0 & \infty \\
\infty & 8 & 0 \\
0 & 0 & 0 \\
0 & 0 & 0\end{array}\right|$ & ฉ & 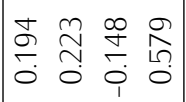 & 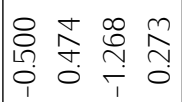 & 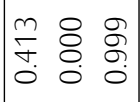 & 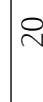 \\
\hline 訔 & 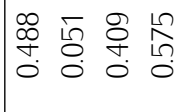 & 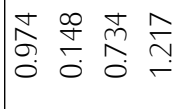 & 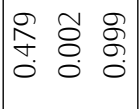 & 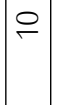 & 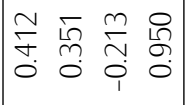 & 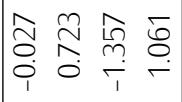 & $\begin{array}{ccc}1 & 0 & \infty \\
0 & 8 & 9 \\
0 & 0 & 5 \\
0 & 0 & 0\end{array} \mid$ & 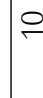 \\
\hline$\stackrel{\square}{\overline{0}}$ & 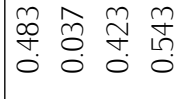 & 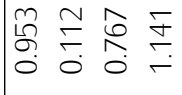 & 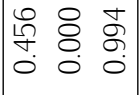 & 우 & 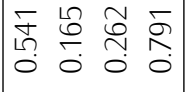 & 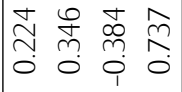 & 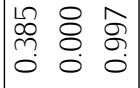 & f \\
\hline & 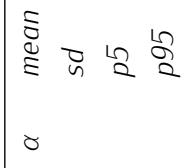 & 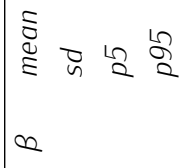 & 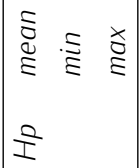 & 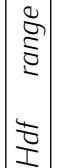 & 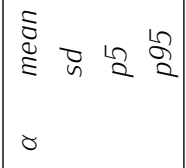 & 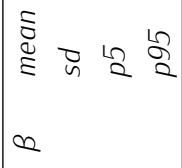 & 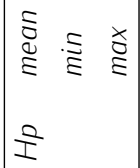 & $\begin{array}{l}8 \\
\vdots \\
\vdots \\
\vdots \\
\vdots\end{array}$ \\
\hline & \multicolumn{4}{|c|}{ 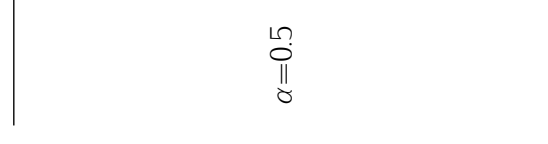 } & \multicolumn{4}{|c|}{$\begin{array}{l}\text { Ln } \\
0 \\
0 \\
0 \\
0\end{array}$} \\
\hline
\end{tabular}




\begin{tabular}{|c|c|c|c|c|c|c|c|c|}
\hline $\begin{array}{l}5 \\
\vdots \\
\vdots \\
n\end{array}$ & 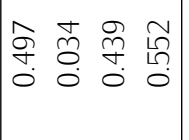 & 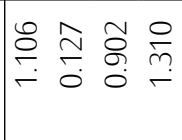 & 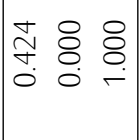 & $\stackrel{\nabla}{\check{1}}$ & 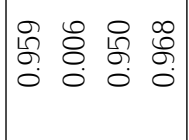 & 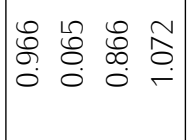 & 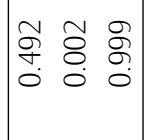 & $\stackrel{m}{?}$ \\
\hline $\begin{array}{l}\stackrel{8}{\geq} \\
\substack{0 \\
n}\end{array}$ & 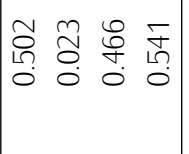 & 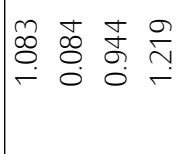 & $\left|\begin{array}{lll}0 & 8 & \infty \\
\multirow{\tau}{*}{} & 8 & 9 \\
0 & 0 & 0 \\
0 & 0 & 0\end{array}\right|$ & ฉ & 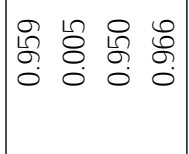 & 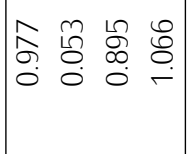 & 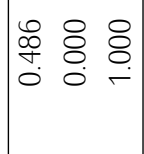 & ๙ \\
\hline$\frac{\vec{n}}{\tilde{n}}$ & 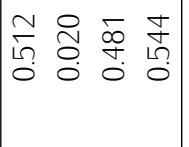 & 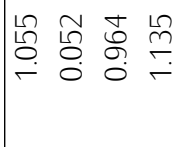 & $\left|\begin{array}{ccc}1 & 0 & 9 \\
\infty & 8 & 8 \\
m & 8 & 9 \\
0 & 0 & 0\end{array}\right|$ & $\approx$ & 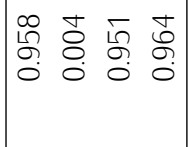 & 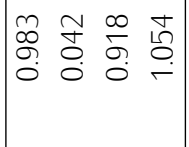 & $\mid \begin{array}{lll}+ & 0 & 8 \\
\infty & 8 & 8 \\
0 & 0 & -\end{array}$ & $\approx$ \\
\hline$\frac{u}{n}$ & 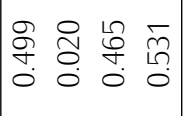 & 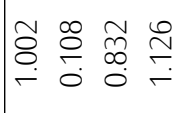 & $\left|\begin{array}{ccc}0 & 8 & 8 \\
\multirow{f}{*}{} & 8 & 8 \\
0 & 0 & \ddots \\
& 0\end{array}\right|$ & $\approx$ & 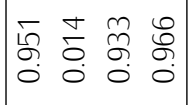 & 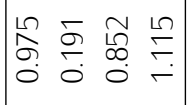 & $\begin{array}{lll}\qquad & 8 & 8 \\
\sigma & 0 & 8 \\
0 & 0 & 0\end{array}$ & $\approx$ \\
\hline$\frac{n}{n}$ & 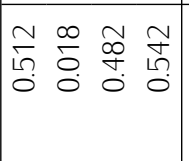 & 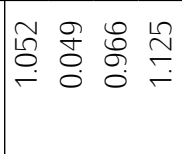 & 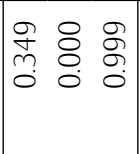 & กิ & 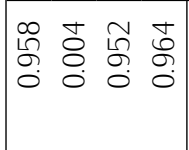 & 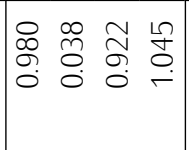 & $\left|\begin{array}{lll}1 & 8 & 9 \\
0 & 8 & 5 \\
0 & 0 & 0 \\
0 & 0 & 0\end{array}\right|$ & in \\
\hline $\begin{array}{l}5 \\
\vdots \\
0 \\
0\end{array}$ & 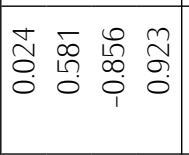 & 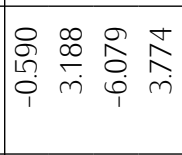 & $\mid \begin{array}{lll}\hat{n} & \overline{8} & g \\
\hat{n} & \delta & \delta \\
0 & 0 & \delta\end{array}$ & $m$ & 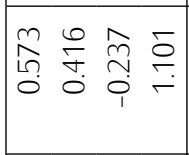 & 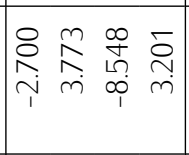 & 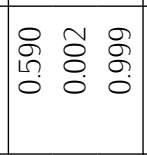 & m \\
\hline 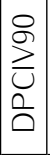 & $\left|\begin{array}{llll}\Re & 0 & 0 & 0 \\
o & m & \infty & 0 \\
0 & \sigma & \sigma & 0 \\
0 & 0 & 0\end{array}\right|$ & 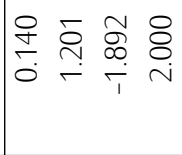 & 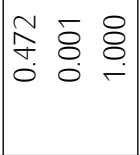 & $\infty$ & 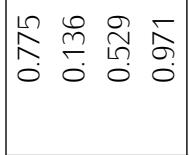 & 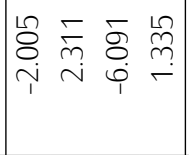 & $\begin{array}{lll}\approx & 8 & 8 \\
1 & 8 & 8 \\
0 & 0 & \ddots\end{array}$ & $\infty$ \\
\hline$\frac{\overline{\bar{D}}}{\overline{0}}$ & 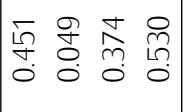 & 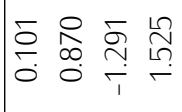 & 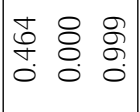 & 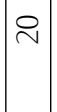 & 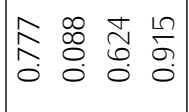 & 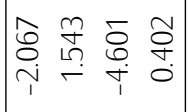 & 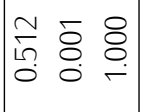 & $\curvearrowright$ \\
\hline $\begin{array}{l}\stackrel{u}{\bar{\Delta}} \\
\end{array}$ & 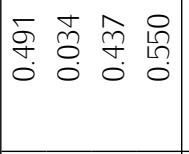 & 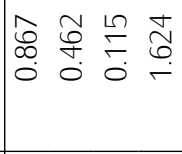 & 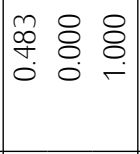 & $\circ$ & 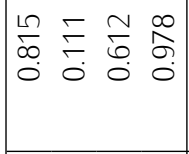 & 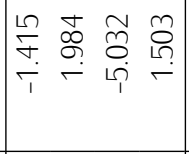 & 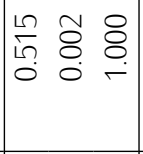 & 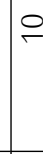 \\
\hline 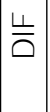 & 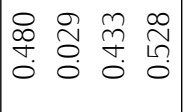 & 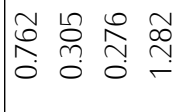 & $\left|\begin{array}{lll}\mathscr{g} & 8 & 1 \\
\forall & 8 & \mathscr{g} \\
0 & 0 & 0 \\
0 & 0 & 0\end{array}\right|$ & 우 & 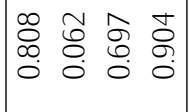 & 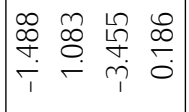 & 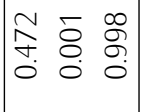 & 웅 \\
\hline & 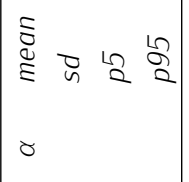 & 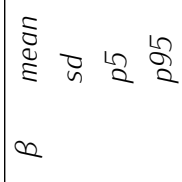 & 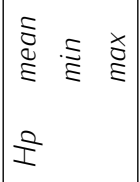 & 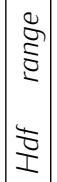 & 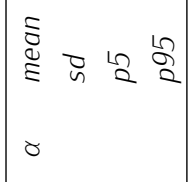 & 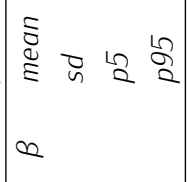 & 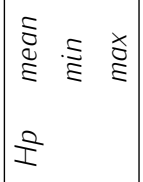 & $\begin{array}{l}8 \\
\vdots \\
\vdots \\
\vdots \\
\vdots\end{array}$ \\
\hline & & $\stackrel{\substack{n \\
0 \\
0}}{\|}$ & & & & & & \\
\hline
\end{tabular}




\begin{tabular}{|c|c|c|c|c|c|c|c|c|}
\hline 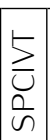 & 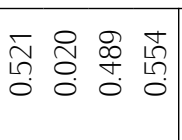 & 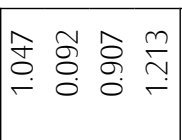 & 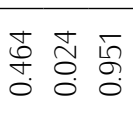 & $\begin{array}{c}\frac{10}{2} \\
\frac{1}{6}\end{array} \mid$ & 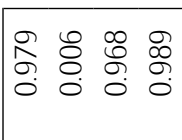 & 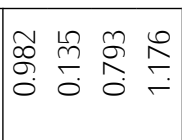 & 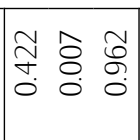 & 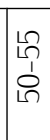 \\
\hline $\begin{array}{l}8 \\
\stackrel{D}{\geq} \\
\substack{n \\
n}\end{array}$ & 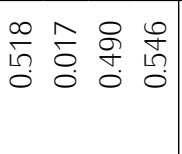 & 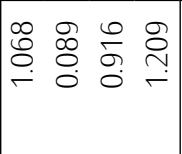 & 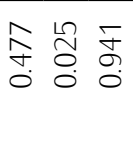 & $\mid \begin{array}{l}0 \\
\infty \\
i \\
N\end{array}$ & $\mid \begin{array}{llll}\infty & 1 & 0 & \\
\vdots & 8 & 8 & \infty \\
\vdots & 0 & 0 & 0 \\
0 & 0 & 0 & 0\end{array}$ & 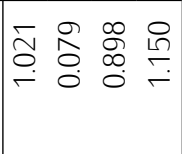 & 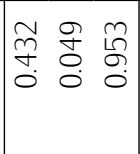 & $\begin{array}{l}\frac{1}{1} \\
\infty \\
\infty \\
\infty \\
\end{array}$ \\
\hline$\frac{\bar{n}}{\omega}$ & 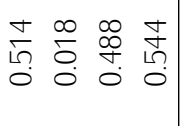 & 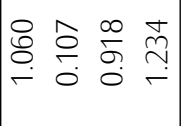 & 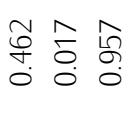 & $\stackrel{+}{\odot}$ & 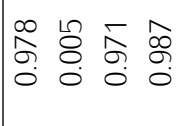 & 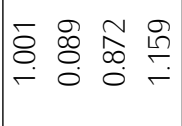 & $\mid \begin{array}{lll}0 & 0 & \infty \\
& \vdots & 0 \\
0 & 0 & 0 \\
0 & 0 & 0\end{array}$ & $\stackrel{5}{\circ}$ \\
\hline $\begin{array}{c}n \\
\vdots \\
n\end{array}$ & 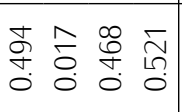 & 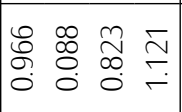 & $\begin{array}{ccc}0 & 0 \\
i n & 0 & 0 \\
0 & 0 & 0 \\
0 & 0\end{array}$ & $\stackrel{\circ}{m}$ & 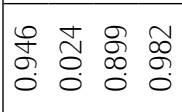 & 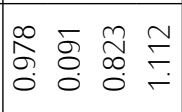 & 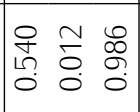 & $\stackrel{m}{m}$ \\
\hline$\frac{n}{n}$ & 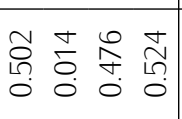 & 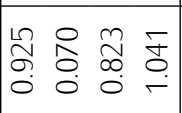 & \begin{tabular}{lll}
$\bar{\sigma}$ & $\bar{\sigma}$ & \multicolumn{1}{c}{} \\
$\bar{\sigma}$ & $\infty$ & 0 \\
0 & 0 & 0 \\
0
\end{tabular} & $\stackrel{\circ}{m}$ & 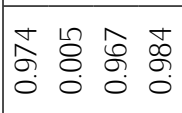 & $\mid \begin{array}{llll}\bar{\sigma} & \bar{\delta} & \bar{m} & \bar{\sigma} \\
\sigma & 0 & 0 & 0 \\
0 & 0 & 0\end{array}$ & 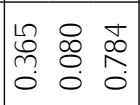 & $\stackrel{\circ}{m}$ \\
\hline $\begin{array}{l}\sum_{0} \\
\vdots \\
0\end{array}$ & 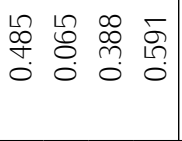 & 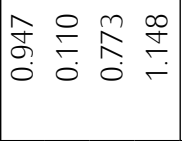 & 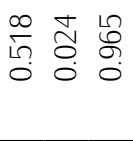 & 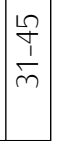 & 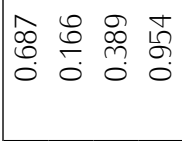 & 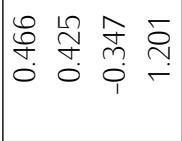 & $\mid \begin{array}{lll}\stackrel{1}{n} & 0 & 0 \\
& \delta & 0 \\
& 0 & 0 \\
0 & 0 & 0\end{array}$ & 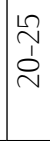 \\
\hline $\begin{array}{l}8 \\
\stackrel{2}{ \pm} \\
\vdots \\
0\end{array}$ & 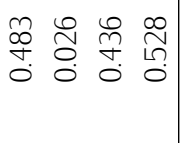 & 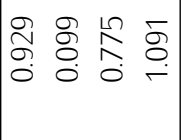 & 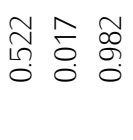 & 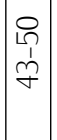 & 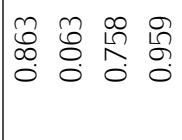 & 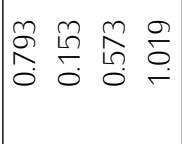 & 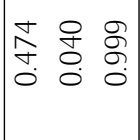 & $\begin{array}{l}0 \\
+ \\
1 \\
+\end{array}$ \\
\hline $\overrightarrow{\overline{0}}$ & 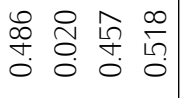 & 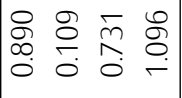 & 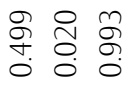 & $\infty$ & 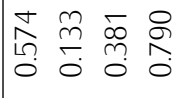 & 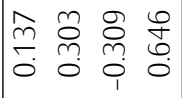 & 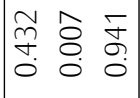 & $\infty$ \\
\hline 訔 & 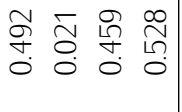 & 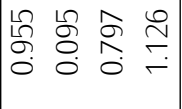 & 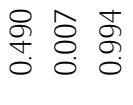 & $m$ & 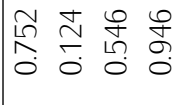 & 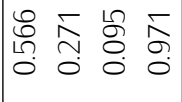 & 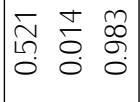 & $m$ \\
\hline 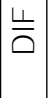 & 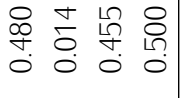 & 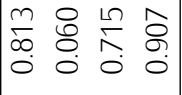 & $\begin{array}{lll}0 & 1 & 0 \\
m & 0 & 0 \\
0 & 0 & 0 \\
0 & 0 & 0 \\
0\end{array}$ & p & 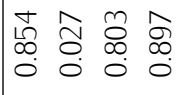 & $\mid \begin{array}{llll} & 0 & 1 & 1 \\
0 & 0 & 0 \\
0 & 0 & 0 \\
0 & 0 & 0 & 0 \\
0 & 0 & 0\end{array}$ & $\mid \begin{array}{ccc}\hat{m} & \infty & 0 \\
& 0 & 0 \\
0 & 0 & 0 \\
0 & 0 & 0\end{array}$ & 吕 \\
\hline & 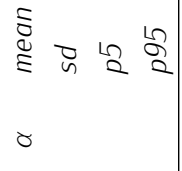 & 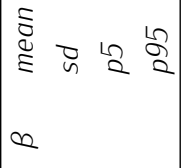 & 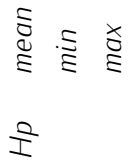 & 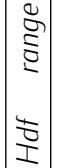 & 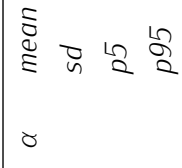 & 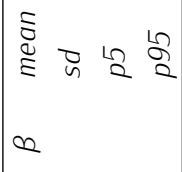 & 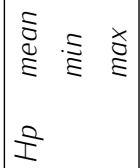 & $\mid \begin{array}{l}8 \\
5 \\
\vdots \\
\vdots \\
\vdots \\
\vdots\end{array}$ \\
\hline & \multicolumn{4}{|c|}{ 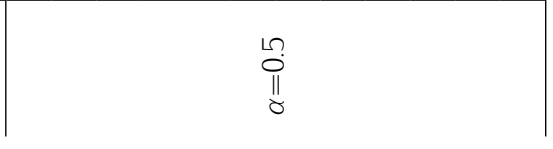 } & \multicolumn{4}{|c|}{$\begin{array}{l}\mathscr{L} \\
0 \\
0 \\
\| \\
0\end{array}$} \\
\hline
\end{tabular}




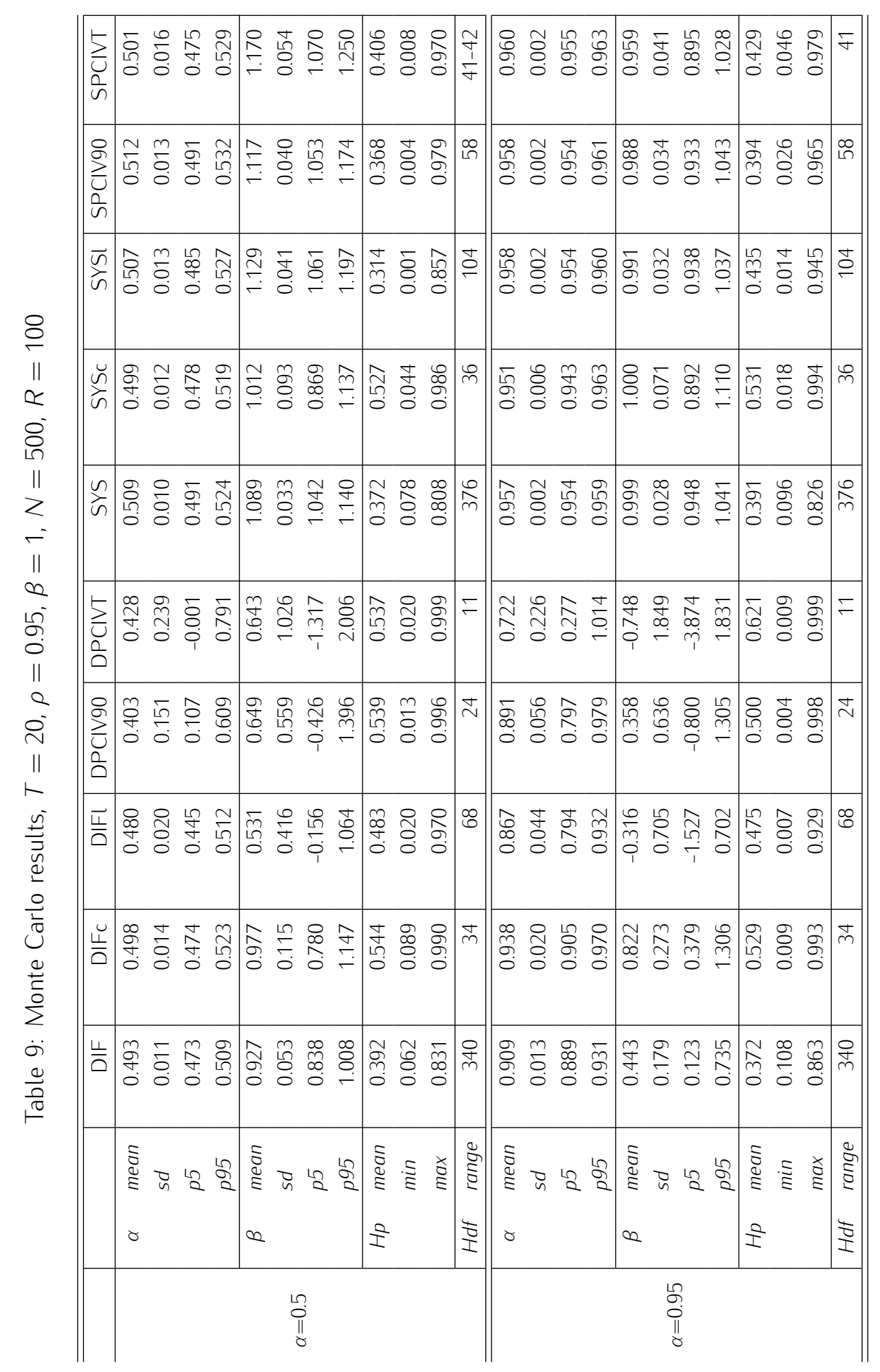


Table 10: Production function: sample size

\begin{tabular}{r|rrr||r|rrr}
\hline \hline Year & Serv. & Manuf. & Total & Year & Serv. & Manuf. & Total \\
\hline 1982 & 5,146 & 10,122 & 15,268 & 1997 & 14,075 & 15,749 & 29,824 \\
1983 & 5,101 & 9,553 & 14,654 & 1998 & 13,786 & 15,398 & 29,184 \\
1984 & 6,371 & 11,421 & 17,792 & 1999 & 14,251 & 15,532 & 29,783 \\
1985 & 7,286 & 12,288 & 19,574 & 2000 & 14,394 & 15,331 & 29,725 \\
1986 & 8,084 & 12,999 & 21,083 & 2001 & 14,138 & 14,456 & 28,594 \\
1987 & 8,490 & 13,225 & 21,715 & 2002 & 13,276 & 13,716 & 26,992 \\
1988 & 9,044 & 13,420 & 22,464 & 2003 & 16,469 & 16,173 & 32,642 \\
1989 & 9,922 & 14,053 & 23,975 & 2004 & 16,875 & 16,365 & 33,240 \\
1990 & 10,563 & 14,546 & 25,109 & 2005 & 15,929 & 14,824 & 30,753 \\
1991 & 10,421 & 14,389 & 24,810 & 2006 & 15,088 & 13,676 & 28,764 \\
1992 & 10,328 & 14,268 & 24,596 & 2007 & 14,115 & 12,709 & 26,824 \\
1993 & 9,275 & 12,155 & 21,430 & 2008 & 13,226 & 12,136 & 25,362 \\
1994 & 13,216 & 14,259 & 27,475 & 2009 & 11,958 & 11,179 & 23,137 \\
1995 & 11,198 & 12,864 & 24,062 & 2010 & 10,529 & 10,081 & 20,610 \\
\cline { 5 - 7 } 1996 & 8,111 & 9,966 & 18,077 & Total & 330,665 & 386,853 & 717,518 \\
\hline \hline
\end{tabular}


Table 11: Production function: statistics

\begin{tabular}{|l|rrrrrrrrr|}
\hline \hline & mean & $p 50$ & \multicolumn{1}{c}{$s d$} & iqr & between & within & residual & $N$ & \multicolumn{1}{c|}{$\bar{T}$} \\
\hline$q_{l}$ & 3.797 & 3.791 & 0.53 & 0.593 & 60.34 & 2.78 & 36.88 & 386853 & 10.13 \\
$c_{l}$ & 3.458 & 3.488 & 1.032 & 1.294 & 79.54 & 3.66 & 16.8 & 284433 & 7.54 \\
$k_{l}$ & 0.215 & 0.246 & 1.537 & 1.931 & 67.17 & 0.34 & 32.49 & 284433 & 7.54 \\
$l$ & 3.908 & 3.829 & 1.06 & 1.242 & 91.59 & 0.62 & 7.8 & 386853 & 10.13 \\
\hline \hline
\end{tabular}

Table 12: Production function: pairwise correlations

\begin{tabular}{|c|c|c|c|c|c|c|}
\hline & $q_{l}$ & $C_{l}$ & $k_{l}$ & l & inv & iinv \\
\hline$q_{l}$ & 1 & & & & & \\
\hline$C_{l}$ & $0.3612^{*}$ & 1 & & & & \\
\hline$k_{l}$ & $0.1622^{*}$ & $0.0941^{*}$ & 1 & & & \\
\hline$l$ & $-0.0978 *$ & $-0.0687^{*}$ & $-0.0626^{*}$ & 1 & & \\
\hline inv & $0.1428^{*}$ & $0.3281^{*}$ & $0.0479 *$ & $-0.0760^{*}$ & 1 & \\
\hline iinv & $0.1114^{*}$ & $0.0311^{*}$ & $0.3425^{*}$ & $-0.0316^{*}$ & $0.1117^{*}$ & 1 \\
\hline
\end{tabular}




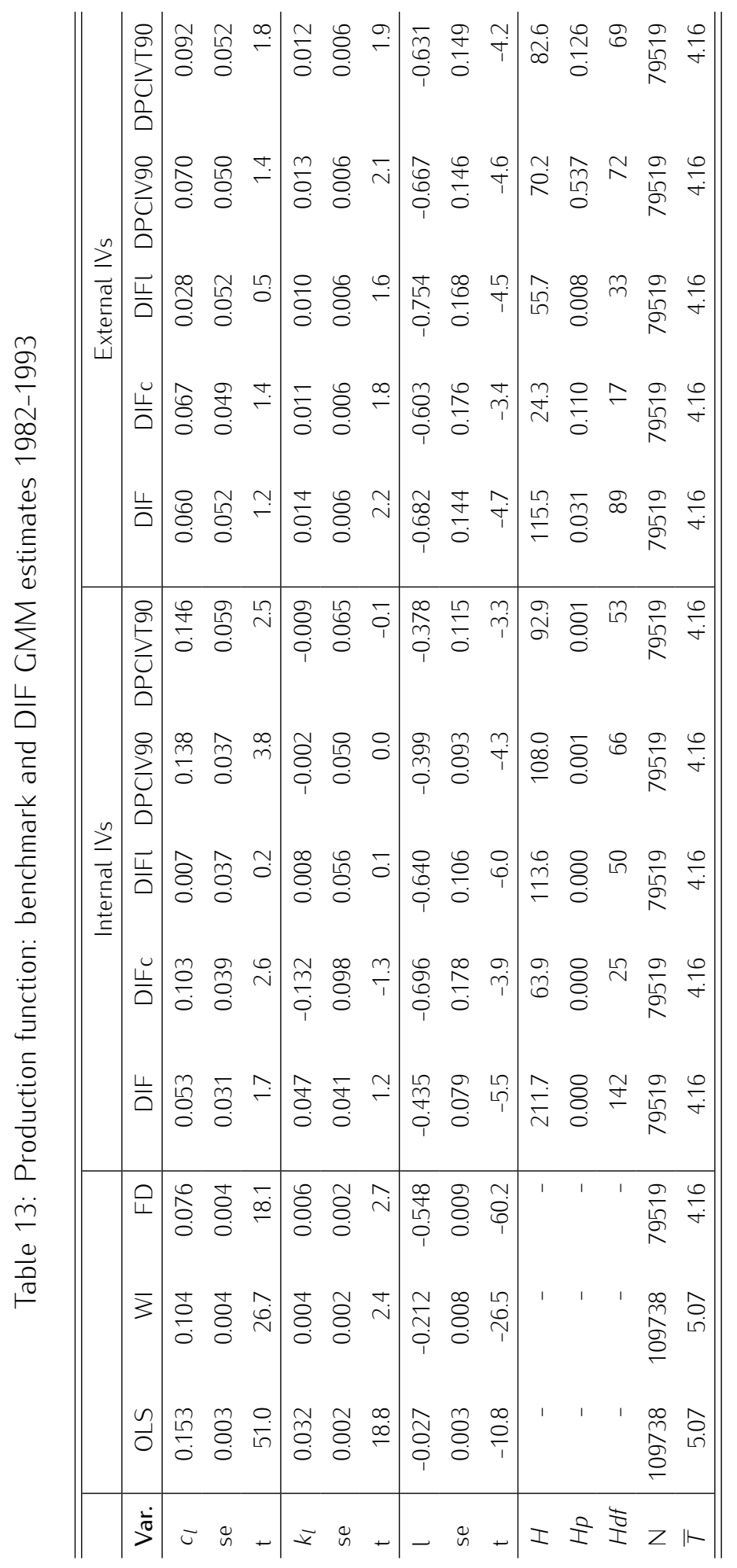




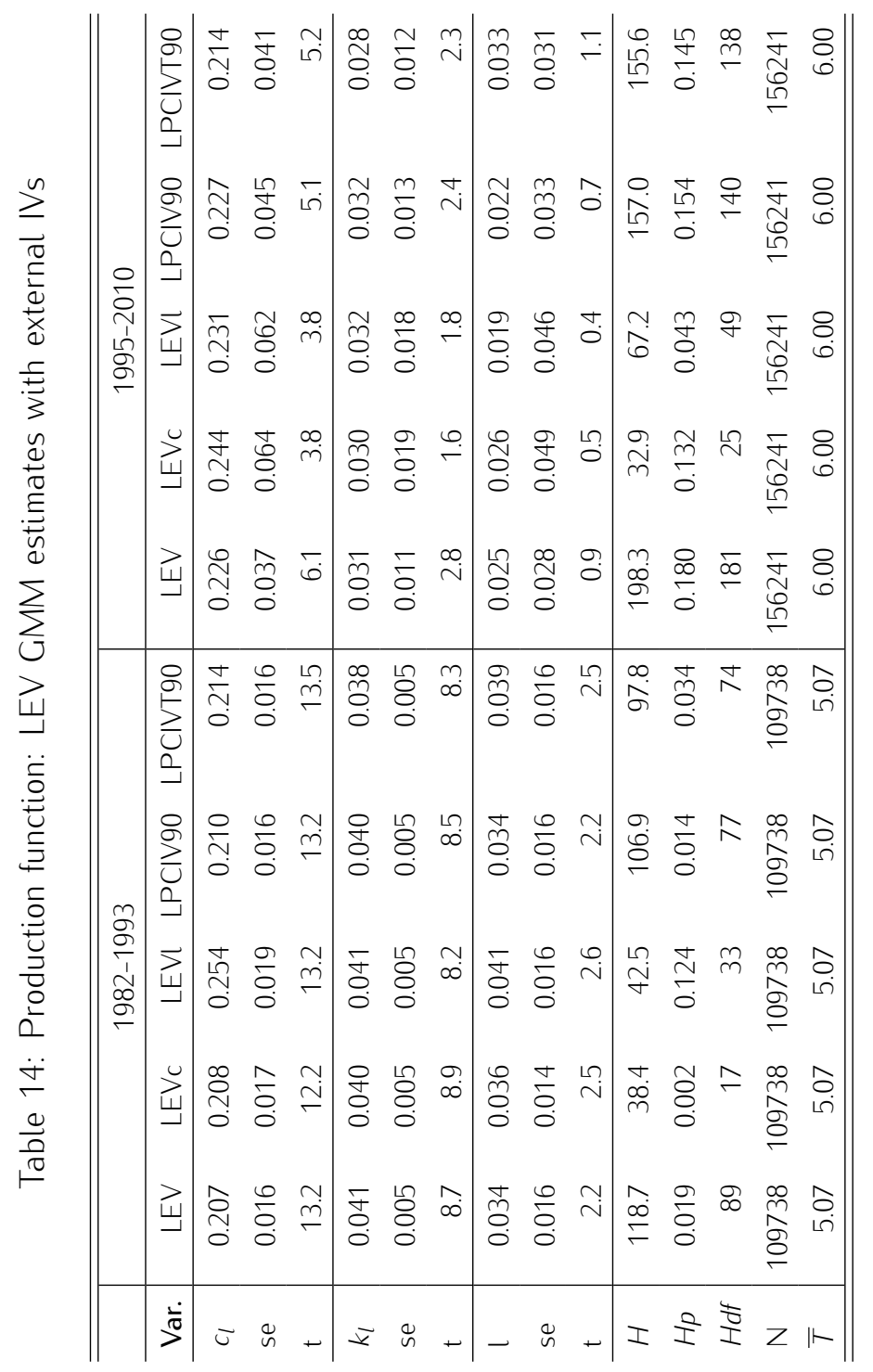




\section{References}

[1] Amemiya, T. (1966) "On the use of principal components of independent variables in two-stage least-squares estimation", International Economic Review, Vol. 7, pp. 283-303.

[2] Arellano, M. (2003) "Modelling optimal instrumental variables for dynamic panel data models", CEMFI, working paper 0310, Madrid.

[3] Arellano, M. and S.R. Bond (1991) "Some tests of specification for panel data: Monte Carlo evidence and an application to employment equations", Review of Economic Studies, vol. 58, pp. 277-297.

[4] Arellano, M., O. Bover (1995) "Another look at the instrumental variables estimation of error-components models", Journal of Econometrics, vol. 68, pp. 29-51.

[5] Bai, J. and S. Ng (2010) "Instrumental Variable Estimation In A Data Rich Environment", Econometric Theory, Vol. 26, pp. 1577-1606.

[6] Barro, R.J. and X. Sala-i-Martin (2003) "Economic Growth, Second edition", MIT Press, Cambridge.

[7] Blundell, R.W. and S.R. Bond (1998) "Initial Conditions and Moment Restrictions in Dynamic Panel Data Models", Journal of Econometrics, vol. 87, pp. 115-143.

[8] Blundell, R.W., S.R. Bond and F. Windmeijer (2000) "Estimation in dynamic panel data models: improving on the performance of the standard GMM estimator", in Baltagi B. (ed.), Advances in Econometrics, vol. 15, Nonstationary panels, panel cointegration, and dynamic panels, JAI Elsevier Sceince, Amsterdam.

[9] Bond, S. (2002) "Dynamic panel data models: a guide to micro data methods and practice", CEMMAP, Working paper 09/02.

[10] Bond, S. and F. Windmeijer (2002) "Finite sample inference for GMM estimators in linear panel data model", CEMMAP, Working paper 04/02.

[11] Bontempi M.E. and J. Mairesse (2008) "Intangible capital and productivity: an exploration on a panel of Italian manufacturing firms", NBER, Working paper n. 14108. 
[12] Bound, J., Jaeger, D.A. and Baker, R.M. (1995) "Problems with instrumental variables estimation when the correlation between the instruments and the endogenous explanatory variable is weak", Journal of the American Statistica Association, vol. 90, pp. 443-450.

[13] Bowsher, C. G. (2002) "On testing overidentifying restrictions in dynamic panel data models", Economics Letters, vol. 77, pp. 211-220.

[14] Bun M.J.G. and F. Windmeijer (2010) "The weak instrument problem of the system GMM estimator in dynamic panal data models", Econometrics Journal, vol. 13, pp. 95-126.

[15] D'Alessio G. (1989) "Multistep principal components analysis in the study of panel data", in Coppi R. and S. Bolasco (eds.), multiway Data Analysis, pp. 375-381, Elsevier Science Publishers B.V. (North-Holland).

[16] Doran H.E. and Schmidt P. (2006) "GMM estimators with improved finite sample properties using principla components of the weighting matrix, with an application to the dynamic panel data model", Journal of econometrics, Vol. 133, pp. 387-409.

[17] Durlauf, S.N., Johnson, P.A. and J.R.W. Temple (2005) "Growth Econometrics", in P. Aghion and S. N. Durlauf (eds.) Handbook of Economic Growth, Vol. 1A, North-Holland, Amsterdam, pp. 555-677.

[18] Forni, M., M. Hallin, M. Lippi, and L. Reichlin (2000) "The Generalized Factor Model: Identification And Estimation", Review of Economics and Statistics, Vol. 82, pp. 540ï¹/2554.

[19] Forni, M., M. Hallin, M. Lippi, and L. Reichlin (2004) "The Generalized Factor Model: Consistency and Rates", Journal of Econometrics, Vol. 119, pp. 231-255.

[20] Forni, M., M. Hallin, M. Lippi, and L. Reichlin (2005) "The Generalized Dynamic Factor Model: One-Sided Estimation and Forecasting", Journal of the American Statistical Association, Vol. 100, pp. 830-839.

[21] Griliches Z. (1998) "R\&D and productivity. The econometric evidence", The University of Chicago Press.

[22] Griliches Z. and J.A. Hausman (1986) "Errors in variables in panel data", Journal of Econometrics, vol. 31, pp. 93-118. 
[23] Griliches Z. and Mairesse J. (1998) "Production Functions: the Search for Identification" in S. Strom (ed.), Essays in Honour of Ragnar Frisch, Econometric Society Monograph Series, Cambridge University Press, Cambridge, pp. 169-203.

[24] Groen, J.J.J. and G. Kapetanios (2009) "Parsimonious estimation with many instruments", Federal Reserve Bank of New York, Staff Report n. 386.

[25] Hall A.R. and F.P.M. Peixe (2003) "A consistent method for the selection of relevant instruments", Econometric Reviews, Vol. 22, pp. 269-287.

[26] Han C. and P.C.B. Phillips (2006) "GMM with many moment conditions", Econometrica, Vol. 74, pp. 147-192.

[27] Hayakawa, K. (2009) "On the effect of mean-nonstationarity in dynamic panel data model", Journal of Econometrics, Vol. 153, pp. 133-135.

[28] Holtz-Eakin D., Newey W. and Rosen H. (1998) "Estimating vector autoregressions with panel data", Econometrica, Vol. 56, pp. 1371-1395.

[29] Kapetanios, G. and M. Marcellino (2010) "Factor-GMM estimation with large sets of possibly weak instruments manuscript", Computational Statistics and Data Analysis, Vol. 54, pp. 2655-2675.

[30] Kloek, T., and L.B.M. Mennes (1960) "Simultaneous equations estimation based on principal components of predetermined variables", Econometrica, Vol. 28, pp. 45-61.

[31] Mairesse J. and B.H. Hall (1995) "Exploring the relationship between R\&D and productivity in French manufactirung firms", Journal of Econometrics, vol. 65, pp. 263-293.

[32] Mairesse J. and B.H. Hall (1996) "Estimating the productivity of research and development in French and US manufacturing firms: an exploration of simultaneity issues with GMM methods", in K. Wagner and Bart Van Ark (eds.) International Productivity Differences and Their Explanations, Elsevier Science, pp. 285-315.

[33] Mairesse J. and M. Sassenou (1991) "R\&D and productivity: a survey of econometric studies at the firm level", NBER, Working paper n. 3666.

[34] Mammi I., (2011) "Essays in GMM estimation of dynamic panel data models", PhD dissertation, IMT Institute for Advanced Studies, Lucca, Italy. 
[35] Mehrhoff, J., (2009) "A solution to the problem of too many instruments in dynamic panel data GMM", Discussion paper n. 1/2009, Deutsche Bundesbank.

[36] Kloek, T., and L.B.M. Mennes (1960) "Simultaneous equations estimation based on principal components of predetermined variables", Econometrica, Vol. 28, pp. 45-61.

[37] Nickell, S. (1981) "Biases in Dynamic Models with Fixed Effects", Econometrica, Vol. 49, pp. 1417-26.

[38] Roodman, D. (2009) "A Note on the theme of too many instruments", Oxford Bulletin of Economics and Statistics, vol. 71, pp. 135-158.

[39] Stock, J.H. and M.W. Watson, (1998) "Diffusion indexes", NBER, Working Paper 6702.

[40] Stock, J.H. and M.W. Watson, (2002a) "Forecasting using principal components from a large number of predictors", Journal of the American Statistical Association, vol. 97, pp. 1167-1179.

[41] Stock, J.H. and M.W. Watson, (2002b) "Macroeconomic forecasting using diffusion indexes", Journal of Business and Economic Statistics, vol. 20, pp. 147-162.

[42] Stock, J.H. and M.W. Watson, (2010) "Dynamic factor models", Chapter 2 in M. Clements and D. Hendry (eds), Oxford Handbook of Economic Forecasting.

[43] Windmeijer F. (2005) "A finite sample correction for the variance of linear efficient two-step GMM estimators", Journal of Econometric, vol. 126, pp. 25-51.

[44] Ziliak J. P. (1997) "Efficient estimation with panel data when instruments are predetermined: an empirical comparison of moment-condition estimators", Journal of Business and Economic Statistics, vol. 15, pp. 419-431. 


\section{Appendix}

\section{The principal component analysis (PCA)}

The PCA is a statistical tool which is used for data reduction according to a data-driven procedure. Intuitively, what PCA does is to find several orthogonal linear combinations of the original variables ordering them on the basis of the portion of the variance in the original data they account for. A principal component is therefore a linear combination of observed variables that is obtained by exploiting a set of optimal weights for each original variable. The first principal component $(\mathrm{PC})$ will be the linear combination of the original variables that has the largest variance among all the possible linear combinations of the original variables. The second $P C$ will be the linear combination, orthogonal to the first $P C$, that accounts for the largest portion of the residual variance once the first PC has been extracted, and so on. All the principal components taken together contain all the information conveyed by the original data.

In other words, through PCA we aim at reducing the dimension of the data while retaining, at the same time, as much of the original variability in the data as possible.

More formally, if we define $\mathrm{C}$ as the $p \times p$ covariance or correlation matrix of the $p$ original variables in the data, the $k^{\text {th }}$ principal component $\mathbf{p c}_{k}$ for $k=$ $1,2, \ldots, p$ is obtained as

$$
\mathrm{pc}_{k}=\mathrm{u}_{k}^{\prime} \mathrm{x}
$$

where $\mathbf{x}$ is the vector of the $p$ variables in the sample, $\mathbf{u}_{k}$ is the $k^{\text {th }}$ eigenvector of $\mathrm{C}$ corresponding to the $k^{\text {th }}$ largest eigenvalue $\lambda_{k}$ subject to the normalization constraints:

$$
\begin{aligned}
& \mathbf{u}_{\mathbf{k}}{ }^{\prime} \mathbf{u}_{\mathbf{k}}=1 \\
& \mathbf{u}_{\mathbf{k}}{ }^{\prime} \mathbf{u}_{\mathbf{j}}=0 \text { for } i \neq j .
\end{aligned}
$$

$\mathrm{pc}_{1}=\mathbf{u}_{1}^{\prime} \mathbf{x}$ is therefore the linear combination of the $p$ variables orthogonal to all other combinations that, subject to the above constraints, has the maximum variance. Similarly $\mathrm{pc}_{2}$ is the linear combination, orthogonal to $p c_{1}$, that maximizes the residual variance.

In matrix notation, we can interpret the principal components in the light of the eigenvalue-eigenvector decomposition of the correlation or the covariance matrix C:

$$
\mathrm{C}=\mathrm{V} \wedge \mathrm{V}^{\prime}=\sum_{i=1}^{p} \lambda_{i} \mathbf{v}_{i} \mathbf{v}_{i}^{\prime}
$$


where $\mathrm{V}$ is the matrix consisting of the eigenvectors (principal components) of $\mathrm{C}, \Lambda$ is the diagonal matrix that has as element $k k$ the eigenvalue $\lambda_{k}$ corresponding to the eigenvector $v_{k}$. The elements $v_{k j}$ of the eigenvector $v_{k}$, namely the coefficients of each linear combination, are the loadings, that represent the contribution of each original value to the PC: in other words, they can be interpreted as the weights of the $j^{\text {th }}$ variable in $\mathbf{p c}_{k}$.

Subject to the conditions in equations (23) and (13), that is if $\mathbf{u}_{k}$ is such to have unit length, the variance of the $k^{\text {th }}$ principal component, $\operatorname{var}\left(\mathbf{p c}_{k}\right)$, is given by $\lambda_{k}$. The total variance of all the principal components will be equal to the variance of the original variables so that:

$$
\sum_{k=1}^{p} \lambda_{k}=\operatorname{tr}(\mathrm{C}) \text {. }
$$

As a consequence, each principal component will account for a portion of the variance of the original data equal to:

$$
P_{k}=\frac{\lambda_{k}}{\operatorname{tr}(\mathrm{C})}
$$

By multiplying each original variable by its loading in each PC, we obtain the matrix of the principal component scores defined as follows:

$$
\mathrm{S}=\mathrm{XV}
$$

where $\mathrm{X}$ is the original data matrix and $\mathrm{V}$ is the same as above. In other terms, the scores $s_{j}$ indicate the influence of a PC on a specific sample. The matrix $\mathrm{S}$ can be used in the analysis in the place of $X$ : in fact, the matrix $\mathbf{S}$ contains the original data matrix in a rotated coordinate system. Clearly the original matrix of data can be written as:

$$
\mathrm{X}=\mathrm{V}^{\prime} \mathrm{S}
$$

where $\mathrm{V}$ and $\mathrm{S}$ are orthogonal.

The number of eigenvalues and eigenvectors, and thus of the principal components, obviously equals the number of variables in the original data.

As the aim of PCA is a reduction of the data dimension through a maximization of the variance explained by the first components and the elimination of multicollinearities in the data, that imply potential problems in inverting the original matrix, we will want to select and keep a number of components $q$ which is smaller than $p$ : we will therefore select the $q$ eigenvectors corresponding to 
the $q$ largest eigenvalues of $\mathbf{C}$ such that they explain most of the variability in the data. The $q$ largest principal components will account for the following portion of the original variance:

$$
\frac{\sum_{k=1}^{q} \lambda_{k}}{\operatorname{tr}(\mathbf{C})}
$$

Accordingly, in the matrix $\mathrm{V}$ only $q$ eigenvectors will be retained and the scores will be computed form the reduced $\mathrm{V}$ matrix.

It is then possible to exploit directly the scores from the PCA by using them instead of the original variables.

A relevant issue is how to choose the the $q$ principal components to be retained in the analysis. Two criteria are generally adopted in the literature: the first implies that only the components that explain a given predetermined portion, usually between $70 \%$ and $90 \%$, of the original variance are to be retained; the second one keeps only the components whose eigenvalues are larger than the average eigenvalue which obviously is the average variance in the original data. 


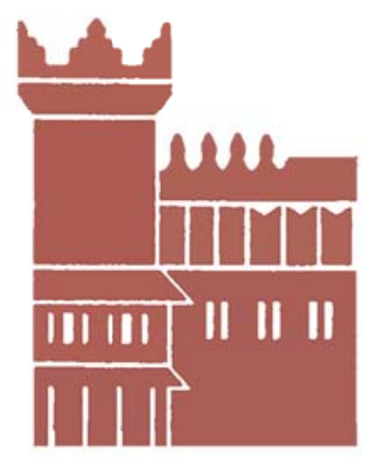

Alma Mater Studiorum - Università di Bologna DEPARTMENT OF ECONOMICS

Strada Maggiore 45

40125 Bologna - Italy

Tel. +39051 2092604

Fax +390512092664

http://www.dse.unibo.it 